\begin{tabular}{|c|l|}
\hline Title & A nisotropic ground states of the quantum Hall system with currents \\
\hline Author(s) & Tsuda, Kazumi; Maeda, Nobuki; Ishikawa, Kenzo \\
\hline Citation & $\begin{array}{l}\text { Physical Review B, 76/4), 045334 } \\
\text { https://doi.org/40.1103/PhysRevB.76.045334 }\end{array}$ \\
\hline Issue Date & 2007-07-25 \\
\hline Doc URL & http://hdl.handle.net/2115/29658 \\
\hline Rights & Copyright $\odot 2007$ A merican Physical Society \\
\hline Type & article \\
\hline File Information & PRB76-4.pdf \\
\hline
\end{tabular}

Instructions for use 


\title{
Anisotropic ground states of the quantum Hall system with currents
}

\author{
Kazumi Tsuda, Nobuki Maeda, and Kenzo Ishikawa \\ Department of Physics, Hokkaido University, Sapporo 060-0810, Japan \\ (Received 14 February 2007; revised manuscript received 1 May 2007; published 25 July 2007)
}

\begin{abstract}
Anisotropic states at half-filled higher Landau levels are investigated in the system with a finite electric current. We study the response of the striped Hall state and the anisotropic charge density wave (ACDW) state against the injected current using the effective action. Current distributions and a current dependence of the total energy are determined for both states. With no injected current, the energy of the ACDW state is lower than that of the striped Hall state. We find that the energy of the ACDW state increases faster than that of the striped Hall state as the injected current increases. Hence, the striped Hall state becomes the lower energy state when the current exceeds the critical value. The critical value is estimated at about $0.04-0.07 \mathrm{nA}$, which is much smaller than the current used in the experiments.
\end{abstract}

DOI: 10.1103/PhysRevB.76.045334

PACS number(s): 73.43. $-\mathrm{f}$

\section{INTRODUCTION}

In the two-dimensional (2D) electron system subjected to a strong perpendicular magnetic field, which is called the quantum Hall system, the half-filled states at each Landau level (LL) exhibit much attractive features. Around the halffilled lowest LL, isotropic compressible states, which are widely believed to be the Fermi liquid of composite fermions, have been observed. ${ }^{1,2}$ Around the half-filled second LL, the $5 / 2$ fractionally quantized Hall conductance has been observed. ${ }^{3}$ The $p$-wave Cooper pairing state of composite fermions, which is called the Pfaffian state, has been proposed to explain this state. ${ }^{4,5}$ Around the half-filled third and higher LLs, highly anisotropic states, which have extremely anisotropic longitudinal resistivities and unquantized Hall resistivities, have been found in ultrahigh mobility samples at low temperature. ${ }^{6,7}$ Many theoretical works have been done to study the anisotropic states. ${ }^{8-19}$ In the present paper, we focus on two different Hartree-Fock (HF) states, i.e., a unidirectional charge density wave state, 8,9 which is called a striped Hall state in the present paper, and an anisotropic charge density wave (ACDW) state. ${ }^{19}$

The experimental features of the anisotropic states suggest that the anisotropic states are the striped Hall states. The striped Hall state has the anisotropic Fermi surface, which has an energy gap in one direction and is gapless in the other direction. The anisotropic longitudinal resistivities and the unquantized Hall resistivities are naturally explained by this anisotropic Fermi surface. ${ }^{20,21}$ On the other hand, the ACDW state has energy gaps in both directions so that it is difficult to explain the experiments with the ACDW state. However, the ACDW state has a lower energy than the striped Hall state in the system with no electric current. This has been a contradiction between the experiments and the theories for the anisotropic state.

In the experiments of the anisotropic states, current is injected. This current effect has not been taken into account in the previous calculations of the total energy. MacDonald et al. have studied the injected current effect on the integer quantum Hall system about two decades ago. ${ }^{23}$ They have calculated the current and charge distributions and found that charges accumulate around both edges of the sample with the opposite sign, as expected from the classical Hall effect. ${ }^{23-26}$ The charge accumulation causes the energy enhancement via the Coulomb interaction between charged particles. The same type of energy corrections may exist even in highly correlated quantum Hall states. However, the effect of the injected current on the anisotropic state has not been studied.

In the present paper, we calculate the correlation energies of the striped Hall state and the ACDW state in the system with the injected current, no impurities, and no metallic contacts. It is important to know if the ACDW state has a lower energy even in the system with the injected current. For this purpose, the dependence of the correlation energies on the injected current is studied in detail. Effects of impurities and metallic contacts are ignored in our calculations of the correlation energies since these effects are expected to be small in the experiments of the anisotropic states in the ultrahigh mobility samples and are outside the scope of this work. The effects of the injected current are investigated using the response functions for electromagnetic fields. The current and charge distributions, are determined and the energies of the two states are calculated from these distributions. It is found that the energy of the ACDW state increases faster than that of the striped Hall state as the injected current increases. Hence, the striped Hall state becomes the lower energy state when the current exceeds the critical value. The critical value is estimated at about $0.04-0.07 \mathrm{nA}$, which is much smaller than the current used in the experiments. Our result suggests that the anisotropic states observed in the experiments are the striped Hall states. Hence, the contradiction between the experiments and the theories is resolved.

This paper is organized as follows. In Sec. II, the two HF states, i.e., the striped Hall state and the ACDW state, are constructed in the von Neumann lattice formalism. In Sec. III, electromagnetic response functions of the two HF states are calculated in the long wavelength limit. Using these response functions, we determine the current and charge distributions and calculate the energy corrections due to currents in Sec. IV. A summary is given in Sec. V.

\section{HARTREE-FOCK GROUND STATES ON THE VON NEUMANN LATTICE}

In this section, the striped Hall state and the ACDW state are constructed in the HF approximation using the von Neu- 
mann lattice base. The von Neumann lattice base is suitable for studying spatially periodic states. We first review the von Neumann lattice base for the completeness of the present paper.

\section{A. von Neumann lattice base}

Let us consider the $2 \mathrm{D}$ electron system in a uniform external magnetic field $B=(\nabla \times \mathbf{A})_{z}$. The spin degree of freedom is ignored, and the natural unit $(\hbar=c=1)$ is used in the present paper. We introduce two sets of coordinates, i.e., the relative coordinates $\xi=(\xi, \eta)$ and the guiding center coordinates $\mathbf{X}=(X, Y)$ :

$$
\begin{gathered}
\xi=\frac{1}{e B}\left(-i \partial_{y}+e A_{y}\right), \quad \eta=-\frac{1}{e B}\left(-i \partial_{x}+e A_{x}\right), \\
X=x-\xi, \quad Y=y-\eta,
\end{gathered}
$$

where $e>0$. Each set of coordinates satisfies the canonical commutation relations

$$
\begin{gathered}
{[X, Y]=-[\xi, \eta]=i / e B,} \\
{[X, \xi]=[X, \eta]=[Y, \xi]=[Y, \eta]=0 .}
\end{gathered}
$$

Using these variables, the one-particle free Hamiltonian is written in the form

$$
H_{0}=\frac{1}{2} m \omega_{c}^{2}\left(\xi^{2}+\eta^{2}\right)
$$

where $\omega_{c}=e B / m$ is a cyclotron frequency. Since $H_{0}$ is equivalent to the Hamiltonian of a harmonic oscillator, the eigenvalue splits into each LL as follows:

$$
H_{0}\left|f_{l}\right\rangle=E_{l}\left|f_{l}\right\rangle, \quad E_{l}=\omega_{c}\left(l+\frac{1}{2}\right) \quad(l=0,1,2, \ldots) .
$$

It is convenient to use a discrete set of coherent states of guiding center coordinates,

$$
(X+i Y)\left|\alpha_{m n}\right\rangle=z_{m n}\left|\alpha_{m n}\right\rangle, \quad z_{m n}=a\left(r_{s} m+i \frac{n}{r_{s}}\right),
$$

where $m$ and $n$ are integers. The completeness of the set $\left\{\left|\alpha_{m n}\right\rangle\right\}$ is ensured, ${ }^{27,28}$ and this set is called the von Neumann lattice (vNL) base. ${ }^{29}$ These coherent states are localized at the rectangular lattice point $a\left(m r_{s}, n / r_{s}\right)$, where a positive real number $r_{s}$ is an asymmetry parameter of the unit cell and $a=\sqrt{2 \pi / e B}$ is a lattice constant. We set $a=1$ unless otherwise stated. Note that the number of lattice points is equal to the number of states in one LL. By Fourier transforming these states, we obtain the orthonormal basis in the momentum representation,

$$
\begin{gathered}
\left|\beta_{\mathbf{p}}\right\rangle=\sum_{m, n} e^{i p_{x} m+i p_{y} n}\left|\alpha_{m n}\right\rangle / \beta(\mathbf{p}), \\
\beta(\mathbf{p})=\left(\sqrt{2} r_{s}\right)^{1 / 2} e^{-\left(r_{s} p_{y}\right)^{2} / 4 \pi} \vartheta_{1}\left(\frac{p_{x}+i r_{s}^{2} p_{y}}{2 \pi} \mid i r_{s}^{2}\right),
\end{gathered}
$$

$$
\left\langle\beta_{\mathbf{p}} \mid \beta_{\mathbf{p}^{\prime}}\right\rangle=\sum_{\mathbf{N}}(2 \pi)^{2} \delta^{2}\left(\mathbf{p}-\mathbf{p}^{\prime}-2 \pi \mathbf{N}\right) e^{i \phi\left(\mathbf{p}^{\prime}, \mathbf{N}\right)},
$$

where $\vartheta_{1}$ is a Jacobi's theta function of the first kind, $\mathbf{N}$ $=\left(N_{x}, N_{y}\right)$ is a vector with integer values, and $\phi(\mathbf{p}, \mathbf{N})$ $=\pi\left(N_{x}+N_{y}\right)-p_{x} N_{y}$. The two-dimensional lattice momentum $\mathbf{p}$ is defined in the Brillouin zone (BZ), $\left|p_{i}\right|<\pi$, and $\beta(\mathbf{p})$ obeys a nontrivial boundary condition

$$
\beta(\mathbf{p}+2 \pi \mathbf{N})=e^{i \phi(\mathbf{p}, \mathbf{N})} \beta(\mathbf{p}) .
$$

The Hilbert space of a one-particle state is spanned by the state $|l, \mathbf{p}\rangle=\left|f_{l}\right\rangle \otimes\left|\beta_{\mathbf{p}}\right\rangle$. We use this base throughout our calculation.

The electron field operator is expanded by the vNL base as

$$
\Psi(\mathbf{x})=\sum_{l=0}^{\infty} \int_{\mathrm{BZ}} \frac{d^{2} p}{(2 \pi)^{2}} b_{l}(\mathbf{p})\langle\mathbf{x} \mid l, \mathbf{p}\rangle,
$$

where $b_{l}(\mathbf{p})$ obeys the same boundary condition as Eq. (2.7) and satisfies the following anticommutation relation:

$$
\left\{b_{l}(\mathbf{p}), b_{l^{\prime}}^{\dagger}\left(\mathbf{p}^{\prime}\right)\right\}=\delta_{l, l^{\prime}} \sum_{\mathbf{N}}(2 \pi)^{2} \delta^{2}\left(\mathbf{p}-\mathbf{p}^{\prime}-2 \pi \mathbf{N}\right) e^{i \phi(\mathbf{p}, \mathbf{N})} .
$$

The Fourier transform of the density operator $\rho(\mathbf{k})$ $=\int d^{2} x e^{i \mathbf{k} \cdot \mathbf{x}} \Psi^{\dagger}(\mathbf{x}) \Psi(\mathbf{x})$ is written as

$$
\rho(\mathbf{k})=\sum_{l, l^{\prime}} \int_{\mathrm{BZ}} \frac{d^{2} p}{(2 \pi)^{2}} b_{l}^{\dagger}(\mathbf{p}) b_{l^{\prime}}(\mathbf{p}-\hat{\mathbf{k}}) f_{l, l^{\prime}}^{0}(\mathbf{k}) e^{-(i / 4 \pi) \hat{k}_{x}\left(2 p_{y}-\hat{k}_{y}\right)},
$$

where $f_{l, l^{\prime}}^{0}(\mathbf{k})=\left\langle f_{l}\left|e^{i \mathbf{k} \cdot \xi}\right| f_{l^{\prime}}\right\rangle \quad($ see Appendix $\quad$ C) and $\hat{\mathbf{k}}$ $=\left(r_{s} k_{x}, k_{y} / r_{s}\right)$.

For a strong magnetic field, in which the energy difference between the nearest LLs, $\omega_{c}$, is much larger than the typical order of the Coulomb interaction, $e^{2} / 4 \pi \epsilon l_{B}$, where $\epsilon$ is the dielectric constant and $l_{B}=\sqrt{1 / e B}$ is the magnetic length, the LL mixing effects can be neglected. In this case, the Hamiltonian is projected to the uppermost partially filled LL, and the kinetic term is quenched. The Hamiltonian in this system is given by the projected Coulomb interaction,

$$
\begin{gathered}
H_{\mathrm{int}}^{(l)}=\frac{1}{2} \int \frac{d^{2} k}{(2 \pi)^{2}}: \rho_{l}(\mathbf{k}) V(\mathbf{k}) \rho_{l}(-\mathbf{k}):, \\
V(\mathbf{k})=\frac{2 \pi q^{2}}{k}\left(k \neq 0, q^{2}=\frac{e^{2}}{4 \pi \epsilon}\right), \quad V(0)=0 .
\end{gathered}
$$

In Eq. (2.11), the colons represent a normal ordering with respect to creation and annihilation operators, $l$ denotes the uppermost partially filled LL index, and $\rho_{l}(\mathbf{k})$ is given by

$$
\begin{gathered}
\rho_{l}(\mathbf{k})=f_{l, l}^{0}(\mathbf{k}) \bar{\rho}_{l}(\mathbf{k}), \\
f_{l, l}^{0}(\mathbf{k})=e^{-k^{2} / 8 \pi} L_{l}^{0}\left(\frac{k^{2}}{4 \pi}\right),
\end{gathered}
$$




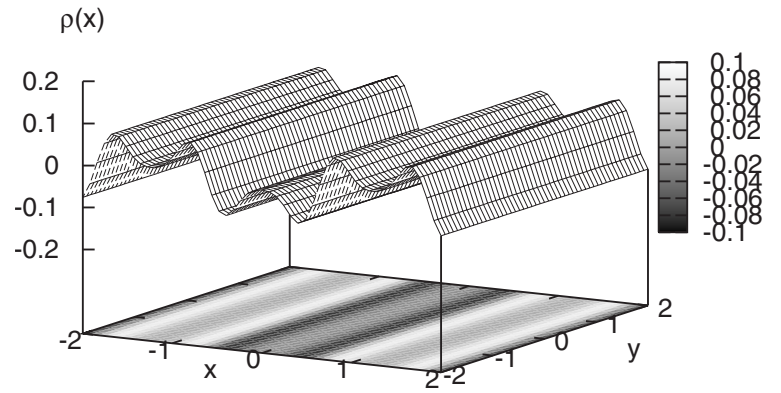

FIG. 1 . Density of the $l=2$ striped Hall state at half-filling. The uniform part $\rho_{0}=\nu^{*}$ is subtracted. The density of the striped Hall state is uniform in the $y$ direction (stripe direction) and periodic with a period $r_{s}$ in the $x$ direction (perpendicular direction).

$$
\bar{\rho}_{l}(\mathbf{k})=\int_{\mathrm{BZ}} \frac{d^{2} p}{(2 \pi)^{2}} b_{l}^{\dagger}(\mathbf{p}) b_{l}(\mathbf{p}-\hat{\mathbf{k}}) e^{-(i / 4 \pi) \hat{k}_{x}\left(2 p_{y}-\hat{k}_{y}\right)},
$$

where $\bar{\rho}_{l}(\mathbf{k})$ is a projected density operator and $L_{l}^{0}$ is a Laguerre polynomial.

$H_{\text {int }}$ is expressed in the HF approximation as (Appendix A) 30,31

$$
H_{\mathrm{HF}}^{(l)}=\mathcal{H}_{\mathrm{HF}}^{(l)}-\frac{1}{2}\left\langle\mathcal{H}_{\mathrm{HF}}^{(l)}\right\rangle,
$$

where

$$
\begin{gathered}
\mathcal{H}_{\mathrm{HF}}^{(l)}=\int \frac{d^{2} k}{(2 \pi)^{2}} v_{l}^{\mathrm{HF}}(\widetilde{\mathbf{k}})\left\langle\bar{\rho}_{l}(-\tilde{\mathbf{k}})\right\rangle \bar{\rho}_{l}(\widetilde{\mathbf{k}}), \\
v_{l}^{\mathrm{HF}}(\mathbf{k})=v_{l}(\mathbf{k})-\int \frac{d^{2} k^{\prime}}{(2 \pi)^{2}} v_{l}\left(\mathbf{k}^{\prime}\right) e^{(i / 2 \pi)\left(k_{x}^{\prime} k_{y}-k_{y}^{\prime} k_{x}\right),} \\
v_{l}(\mathbf{k})=V(\mathbf{k})\left[f_{l, l}^{0}(\mathbf{k})\right]^{2}, \quad \widetilde{\mathbf{k}}=\left(\frac{k_{x}}{r_{s}}, r_{s} k_{y}\right) .
\end{gathered}
$$

This HF Hamiltonian has been diagonalized selfconsistently, and various ground states have been obtained. In the present paper, we concentrate on the striped Hall state and the ACDW state at half-filled higher LLs. These states are constructed using the vNL base in the following subsections.

\section{B. Striped Hall state}

We consider the case of the filling factor $\nu=l+\nu^{*}$, with $\nu^{*}=1 / 2$. The present formalism is valid for the arbitrary $\nu^{*}$ $\left(0<\nu^{*}<1\right)$. The striped Hall state is a unidirectional charge density wave state which has the following unidirectional density (Fig. 1):

$$
\left\langle\rho_{l}(\mathbf{x})\right\rangle_{\text {stripe }}=\sum_{N_{x}} \Delta_{l}\left(N_{x}\right) f_{l, l}^{0}\left(\frac{2 \pi N_{x}}{r_{0}}, 0\right) e^{i\left(2 \pi N_{x} / r_{0}\right) x},
$$

where $r_{0}$ is the period of the density in the $x$ direction, $\Delta_{l}\left(N_{x}\right)$ is an order parameter determined self-consistently, and

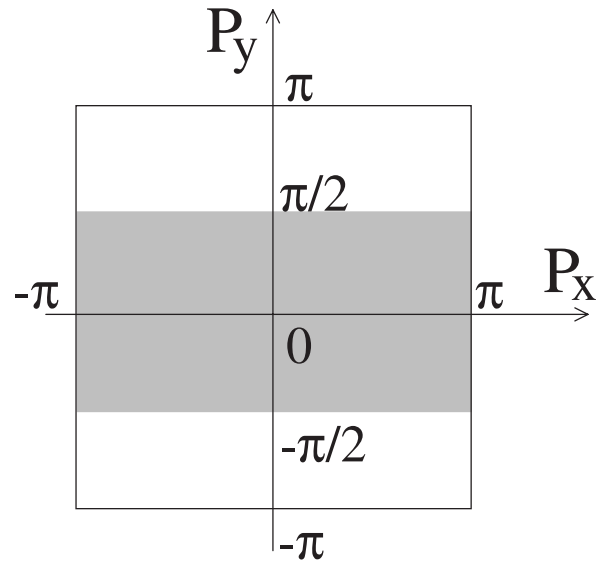

FIG. 2. Fermi sea of the striped Hall state at half-filling. The occupied state is represented by the dark region. When the stripe direction faces the $y$ direction, the $p_{x}$ direction of the Brillouin zone is fully occupied. In this case, the Fermi sea has the inter-LL energy gap in the $p_{x}$ direction and is gapless in the $p_{y}$ direction.

$\Delta_{l}(0)=\nu^{*}$. We call the uniform direction stripe direction and the other direction perpendicular direction in this paper. Equation (2.16) gives the following form of $\left\langle\bar{\rho}_{l}(\mathbf{k})\right\rangle$ :

$$
\left\langle\bar{\rho}_{l}(\mathbf{k})\right\rangle_{\text {stripe }}=\sum_{N_{x}} \Delta_{l}\left(N_{x}\right)(2 \pi)^{2} \delta\left(k_{x}+\frac{2 \pi N_{x}}{r_{0}}\right) \delta\left(k_{y}\right) .
$$

The HF Hamiltonian of the striped Hall state is easily diagonalized on the vNL by taking the asymmetry parameter $r_{s}$ $=r_{0}$. Substituting Eq. (2.17) into the HF Hamiltonian and using $r_{s}=r_{0}$, the HF Hamiltonian of the striped Hall state is written by

$$
\mathcal{H}_{\mathrm{HF} \text { stripe }}^{(l)}=\int_{\mathrm{BZ}} \frac{d^{2} p}{(2 \pi)^{2}} \epsilon_{l}(\mathbf{p}) b_{l}^{\dagger}(\mathbf{p}) b_{l}(\mathbf{p}),
$$

where $\epsilon_{l}(\mathbf{p})$ is a one-particle energy given by

$$
\epsilon_{l}(\mathbf{p})=\epsilon_{0}+\sum_{N_{x} \neq 0} \Delta_{l}\left(N_{x}\right) v_{l}^{\mathrm{HF}}\left(\frac{2 \pi N_{x}}{r_{s}}, 0\right)(-1)^{N_{x}} e^{-i N_{x} p_{y}}
$$

In Eq. (2.19), $\epsilon_{0}$ is a uniform Fock energy given by $\nu^{*} v_{l}^{\mathrm{HF}}(0)$.

From Eq. (2.18), the two-point function of the operator $b_{l}(\mathbf{p})$ is given by ${ }^{20,21}$

$$
\begin{aligned}
\left\langle b_{l}^{\dagger}(\mathbf{p}) b_{l^{\prime}}\left(\mathbf{p}^{\prime}\right)\right\rangle_{\text {stripe }}= & \sum_{\mathbf{N}} \delta_{l, l^{\prime}} \theta\left[\mu_{F}-\epsilon_{l}(\mathbf{p})\right](2 \pi)^{2} \delta^{2}\left(\mathbf{p}-\mathbf{p}^{\prime}\right. \\
& -2 \pi \mathbf{N}) e^{-i \phi(\mathbf{p}, \mathbf{N})}
\end{aligned}
$$

where $\mu_{F}$ is a Fermi energy and $\theta$ is a step function. The self-consistent equation for $\Delta_{l}\left(N_{x}\right)$ is obtained by substituting Eq. (2.20) into the left hand side of Eq. (2.17). $\Delta_{l}\left(N_{x}\right)$ $=(-1)^{N_{x}} \sin \left(\nu^{*} \pi N_{x}\right) / \pi N_{x}$ is a solution of the self-consistent equation. This solution has the Fermi sea, $\left|p_{y}\right|<\pi \nu^{*}$ (shown in Fig. 2) and gives the one-particle energy as (Fig. 3) 


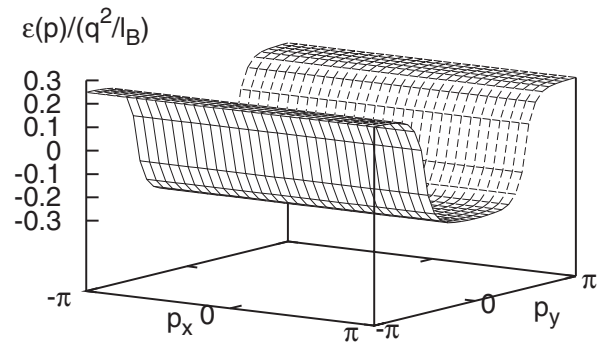

FIG. 3. One-particle energy of the $l=2$ striped Hall state at half-filling. The uniform Fock energy is subtracted. When the stripe direction faces the $y$ direction, the one-particle energy is uniform in the $p_{x}$ direction.

$$
\epsilon_{l}(\mathbf{p})=\epsilon_{0}+\sum_{N_{x} \neq 0} v_{l}^{\mathrm{HF}}\left(\frac{2 \pi N_{x}}{r_{s}}, 0\right) \frac{\sin \left(\nu^{*} \pi N_{x}\right)}{\pi N_{x}} e^{-i N_{x} p_{y}} .
$$

The HF energy per particle is given as a function of $r_{s}$ by

$$
\begin{aligned}
E_{\text {stripe }}^{(l)}\left(r_{s}\right)= & \frac{\left\langle H_{\mathrm{HF}}^{(l)}\right\rangle_{\text {stripe }}}{N_{e}^{(l)}}=\frac{1}{2} \epsilon_{0}+\frac{1}{2} \sum_{N_{x} \neq 0} \nu^{*} v_{l}^{\mathrm{HF}}\left(\frac{2 \pi N_{x}}{r_{s}}, 0\right) \\
& \times\left[\frac{\sin \left(\nu^{*} \pi N_{x}\right)}{\nu^{*} \pi N_{x}}\right]^{2},
\end{aligned}
$$

where $N_{e}^{(l)}$ is the total number of particles within the $l$ th LL. The optimal value of $r_{s}$ is determined by minimizing $E_{\text {stripe }}^{(l)}$ $\times\left(r_{s}\right)$. The optimal value of $r_{s}$ and the minimum energy at each LL are shown in Table I. ${ }^{21}$

The striped Hall state has the anisotropic Fermi surface shown in Fig. 2, which has an inter-LL energy gap in the $p_{x}$ direction and is gapless in the $p_{y}$ direction. This would cause the anisotropic longitudinal resistivities.

\section{Anisotropic charge density wave state}

We consider the ACDW state with the following rectangular charge density wave (Fig. 4): ${ }^{22}$

$$
\left\langle\rho_{l}(\mathbf{x})\right\rangle_{\mathrm{ACDW}}=\sum_{\mathbf{N}} \Delta_{l}\left(\mathbf{Q}_{N}\right) f_{l, l}^{(l)}\left(\mathbf{Q}_{N}\right) e^{-i \mathbf{Q}_{N} \mathbf{x}},
$$

where $\mathbf{Q}_{N}=\left(2 \pi N_{x} / r_{0 x}, 2 \pi N_{y} / r_{0 y}\right)\left(r_{0 x}\right.$ and $r_{0 y}$ are the periods of the density in the $x$ direction and the $y$ direction, respectively), $\Delta_{l}\left(\mathbf{Q}_{N}\right)$ is an order parameter determined selfconsistently, and $\Delta_{l}(0)=\nu^{*}$. We call the direction with a shorter period of the density $A C D W$ direction in this paper.

TABLE I. Minimum energy and corresponding parameter $r_{s}$ of the striped Hall states at $\nu=l+1 / 2$.

\begin{tabular}{llc}
\hline \hline$l$ & $r_{s}^{\text {stripe }}$ & $E_{\text {stripe }} /\left(q^{2} / l_{B}\right)$ \\
\hline 0 & 1.636 & -0.4331 \\
1 & 2.021 & -0.3490 \\
2 & 2.474 & -0.3074 \\
3 & 2.875 & -0.2800 \\
\hline \hline
\end{tabular}

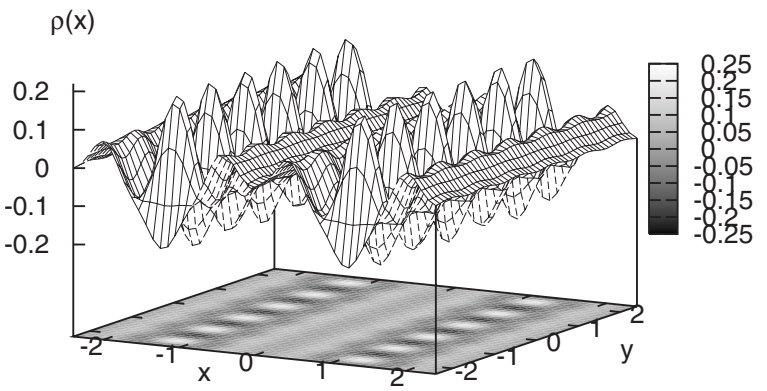

FIG. 4. Density of the $l=2$ ACDW state at half-filling. The uniform part $\rho_{0}=\nu^{*}$ is subtracted. The density of the ACDW state is periodic in the both directions. The $y$ direction is referred to as the ACDW direction in this figure.

Equation (2.23) gives the following form of $\left\langle\bar{\rho}_{l}(\mathbf{k})\right\rangle$ :

$$
\left\langle\bar{\rho}_{l}(\mathbf{k})\right\rangle_{\mathrm{ACDW}}=\sum_{\mathbf{N}} \Delta_{l}\left(\mathbf{Q}_{N}\right)(2 \pi)^{2} \delta^{2}\left(\mathbf{k}-\mathbf{Q}_{N}\right) .
$$

The number of ACDW unit cells $N_{\mathrm{CDW}}=($ area $) / r_{0 x} r_{0 y}$ is equal to the number of electrons within the $l$ th LL, while the number of $\mathrm{vNL}$ unit cells $N_{\mathrm{vNL}}=($ area $) / a^{2}$ is equal to the number of states in one LL. Here, we write the vNL constant $a$ explicitly. Hence, the filing factor $\nu^{*}$ is expressed as

$$
\nu^{*}=\frac{N_{\mathrm{CDW}}}{N_{\mathrm{vNL}}}=\frac{a^{2}}{r_{0 x} r_{0 y}} .
$$

Using the dimensionless lattice parameter $r_{0}$ defined as $r_{0 x}$ $=a r_{0}, \mathbf{Q}_{N}$ is written as

$$
\mathbf{Q}_{N}=\left(\frac{2 \pi N_{x}}{a r_{0}}, \frac{2 \pi N_{y} \nu^{*} r_{0}}{a}\right)
$$

and $\left\langle\bar{\rho}_{l}(\mathbf{k})\right\rangle_{\mathrm{ACDW}}$ is rewritten as

$$
\begin{aligned}
\left\langle\bar{\rho}_{l}(\mathbf{k})\right\rangle_{\mathrm{ACDW}}= & \sum_{\mathbf{N}} \Delta_{l}\left(\frac{2 \pi N_{x}}{r_{0}}, 2 \pi N_{y} \nu^{*} r_{0}\right)(2 \pi)^{2} \\
& \times \delta\left(k_{x}-\frac{2 \pi N_{x}}{r_{0}}\right) \delta\left(k_{y}-2 \pi N_{y} \nu^{*} r_{0}\right),
\end{aligned}
$$

where we set $a=1$ again.

The HF Hamiltonian of the ACDW state is easily diagonalized using the vNL base. We concentrate on the case of $\nu^{*}=1 / 2$. In this case, the ACDW unit cell is just twice as large as the vNL unit cell (Fig. 5). When we take $r_{s}=r_{0}$ and divide $N_{y}$ in the right hand side of Eq. (2.27) into even and odd, the mean value of the projected density operator is rewritten as

$$
\begin{aligned}
\left\langle\bar{\rho}_{l}(\tilde{\mathbf{k}})\right\rangle_{\mathrm{ACDW}}= & \sum_{\mathbf{N}}\left\{\Delta_{l}\left(\frac{2 \pi N_{x}}{r_{s}}, 2 \pi N_{y} r_{s}\right)(2 \pi)^{2} \delta^{2}(\mathbf{k}-2 \pi \mathbf{N})\right. \\
& +\Delta_{l}\left[\frac{2 \pi N_{x}}{r_{s}}, \pi\left(2 N_{y}+1\right) r_{s}\right](2 \pi)^{2} \\
& \left.\delta\left(k_{x}-2 \pi N_{x}\right) \delta\left[k_{y}-\pi\left(2 N_{y}+1\right)\right]\right\}
\end{aligned}
$$




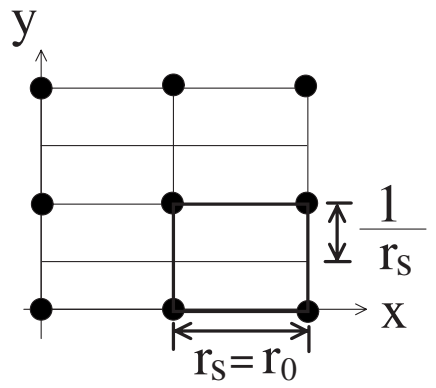

FIG. 5. vNL unit cell and ACDW unit cell. The black circles represent the location of electrons and the thin lines represent the vNL. The ACDW unit cell represented by the bold lines is just twice as large as the vNL unit cell in the case of $\nu^{*}=1 / 2$.

Substituting this expression into Eq. (2.14), the HF Hamiltonian of the ACDW state is obtained by

$$
\begin{aligned}
& \mathcal{H}_{\mathrm{HF} \mathrm{ACDW}}^{(l)}= \epsilon_{0} N_{e}^{(l)}+\int_{\mathrm{BZ}} \frac{d^{2} p}{(2 \pi)^{2}}\left[A(\mathbf{p}) b_{l}^{\dagger}(\mathbf{p}) b_{l}(\mathbf{p})\right. \\
&\left.+B(\mathbf{p}) b_{l}^{\dagger}(\mathbf{p}) b_{l}\left(p_{x}, p_{y}+\pi\right)\right], \\
& A(\mathbf{p})=\sum_{\mathbf{N} \neq 0} v_{l}^{\mathrm{HF}}\left(\frac{2 \pi N_{x}}{r_{s}}, 2 \pi N_{y} r_{s}\right) \Delta_{l}\left(\frac{2 \pi N_{x}}{r_{s}}, 2 \pi N_{y} r_{s}\right) \\
& \times e^{i \pi\left(N_{x}+N_{y}+N_{x} N_{y}\right)-i p_{x} N_{y}+i p_{y} N_{x}}, \\
& B(\mathbf{p})=\sum_{\mathbf{N}} v_{l}^{\mathrm{HF}}\left[\frac{2 \pi N_{x}}{r_{s}}, \pi\left(2 N_{y}+1\right) r_{s}\right] \\
& \times \Delta_{l}\left[\frac{2 \pi N_{x}}{r_{s}}, \pi\left(2 N_{y}+1\right) r_{s}\right] \\
& \times e^{i \pi\left[N_{x}+N_{y}+N_{x}\left(N_{y}+1 / 2\right)\right]-i p_{x} N_{y}+i p_{y} N_{x} .}
\end{aligned}
$$

In this Hamiltonian, momentum is not conserved since $b_{l}^{\dagger}(\mathbf{p})$ is coupled with $b_{l}\left(p_{x}, p_{y}+\pi\right)$. However, using the boundary condition for $b_{l}(\mathbf{p})$, the HF Hamiltonian is rewritten as

$$
\begin{gathered}
\mathcal{H}_{\mathrm{HFACDW}}^{(l)}=\epsilon_{0} N_{e}^{(l)}+\int_{\mathrm{RBZ}} \frac{d^{2} p}{(2 \pi)^{2}} \mathbf{b}_{l}^{\dagger}(\mathbf{p}) D_{l}(\mathbf{p}) \mathbf{b}_{l}(\mathbf{p}), \\
\mathbf{b}_{l}(\mathbf{p})=\left(\begin{array}{c}
b_{l}\left(p_{x}, p_{y}\right) \\
b_{l}\left(p_{x}, p_{y}+\pi\right)
\end{array}\right), \\
D_{l}(\mathbf{p})=\left(\begin{array}{cc}
A(\mathbf{p}) & B(\mathbf{p}) \\
B^{*}(\mathbf{p}) & A\left(p_{x}, p_{y}+\pi\right)
\end{array}\right)
\end{gathered}
$$

where the momentum integration is performed over the reduced Brillouin zone (RBZ), $\left|p_{x}\right|<\pi$ and $\left|p_{y}\right|<\pi / 2$, and $D_{l}(\mathbf{p})$ is a $2 \times 2$ Hermite matrix. The Hamiltonian expressed by Eq. (2.31) can be diagonalized at each momentum just by unitary transforming the field operator $\mathbf{b}_{l}(\mathbf{p})$. In the present case of $\nu^{*}=1 / 2$, the Brillouin zone is reduced to the half size of the original domain, and two energy bands are formed (Fig. 6). $D_{l}(\mathbf{p})$ is diagonalized using the unitary matrix $U(\mathbf{p})$ as

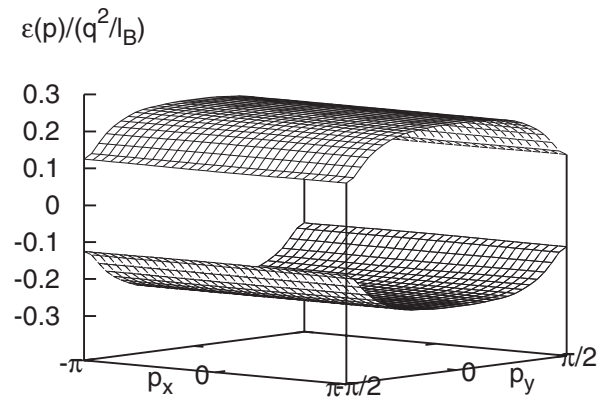

FIG. 6. One-particle energy of the $l=2$ ACDW state at halffilling. The uniform Fock energy is subtracted. Two bands are formed and the lower band is completely filled.

$$
U^{\dagger}(\mathbf{p}) D_{l}(\mathbf{p}) U(\mathbf{p})=\left(\begin{array}{cc}
\epsilon_{+}(\mathbf{p}) & 0 \\
0 & \epsilon_{-}(\mathbf{p})
\end{array}\right)
$$

where $\epsilon_{+}(\mathbf{p})$ and $\epsilon_{-}(\mathbf{p})$ represent the upper energy band and the lower energy band, respectively. $\epsilon_{ \pm}(\mathbf{p})$ and $U(\mathbf{p})$ are given in Appendix B. Using the base $\mathbf{c}_{l}(\mathbf{p})=U^{\dagger}(\mathbf{p}) \mathbf{b}_{l}(\mathbf{p})$, the HF Hamiltonian of the ACDW state is obtained by

$$
\mathcal{H}_{\mathrm{HF} \mathrm{ACDW}}^{(l)}=\epsilon_{0} N_{e}^{(l)}+\int_{\mathrm{RBZ}} \frac{d^{2} p}{(2 \pi)^{2}} \mathbf{c}_{l}^{\dagger}(\mathbf{p})\left(\begin{array}{cc}
\epsilon_{+}(\mathbf{p}) & 0 \\
0 & \epsilon_{-}(\mathbf{p})
\end{array}\right) \mathbf{c}_{l}(\mathbf{p}),
$$

where

$$
\mathbf{c}_{l}(\mathbf{p})=\left(\begin{array}{c}
c_{+}(\mathbf{p}) \\
c_{-}(\mathbf{p})
\end{array}\right) .
$$

$\Delta_{l}\left(\mathbf{Q}_{N}\right)$ is determined by solving the self-consistent equation numerically (see Appendix B).

As in the case of the striped Hall state, the HF energy of the ACDW state also depends on the asymmetry parameter $r_{s}$. The optimal value of $r_{s}$, the HF energy per particle, and the magnitude of the energy gap are given in Table II, in which there are two values of $r_{s}$ at each LL due to the $\pi / 2$-rotational symmetry. The magnitude of the energy gap is estimated to be of the order of $10 \mathrm{~K}$ for a few tesla. Experiments for the anisotropic states have shown the extremely anisotropic longitudinal resistivities and the unquantized Hall resistivities at tens of milliKelvin. It is difficult to explain the experiments with the ACDW state. On the other hand, the HF energy of the ACDW state is slightly lower than that of the striped Hall state at each LL, as seen in Tables I and II. This was one of the remaining issues for the

TABLE II. Minimum energy and corresponding parameter $r_{s}$ of the ACDW states at $\nu=l+1 / 2$. $\Delta_{\text {gap }}$ is a magnitude of the energy gap.

\begin{tabular}{lccc}
\hline \hline$l$ & $r_{s}^{\mathrm{ACDW}}$ & $E_{\mathrm{ACDW}} /\left(q^{2} / l_{B}\right)$ & $\Delta_{\mathrm{gap}} /\left(q^{2} / l_{B}\right)$ \\
\hline 0 & $\sqrt{2}$ & -0.4436 & 0.3292 \\
1 & $1.02,1.96$ & -0.3583 & 0.3077 \\
2 & $0.82,2.44$ & -0.3097 & 0.2470 \\
3 & $0.70,2.86$ & -0.2814 & 0.1967 \\
\hline \hline
\end{tabular}


anisotropic states. In Sec. IV, we study the total energy of the present two HF states in the system with injected currents to answer this issue.

\section{RESPONSE FUNCTIONS}

In this section, the electromagnetic response functions of the HF states are calculated in the long wavelength limit. We consider the quantum Hall system with the infinitesimal external gauge field $a_{\mu}(x)=\left[a_{0}(x),-\boldsymbol{a}(x)\right]$ and calculate the response functions of the striped Hall state first and the ACDW state next.

\section{A. Response function of the striped Hall state}

The Hamiltonian in the quantum Hall system with $a_{\mu}(x)$ is given by

$$
\begin{aligned}
H= & \int d^{2} x \Psi^{\dagger}(x)\left\{\frac{[\mathbf{p}+e \mathbf{A}(x)+e \boldsymbol{a}(x)]^{2}}{2 m}-e a_{0}(x)\right\} \Psi(x) \\
& +\frac{1}{2} \int d^{2} x d^{2} x^{\prime}: \rho(x) V\left(x-x^{\prime}\right) \rho\left(x^{\prime}\right):
\end{aligned}
$$

where $V(x)=q^{2} /|\mathbf{x}|$. We project the Coulomb interaction part to each LL and apply the HF approximation to the projected Coulomb interaction. Then, using the vNL base, the Hamiltonian in the HF approximation is given by

$$
\begin{aligned}
H= & \sum_{l} E_{l} \int_{\mathrm{BZ}} \frac{d^{2} p}{(2 \pi)^{2}} b_{l}^{\dagger}(\mathbf{p}) b_{l}(\mathbf{p})-\int \frac{d^{2} k}{(2 \pi)^{2}} \sum_{l, l^{\prime}} e f_{l, l^{\prime}}^{\mu}(\tilde{\mathbf{k}}) a_{\mu}(\tilde{\mathbf{k}}) \\
& \times \int_{\mathrm{BZ}} \frac{d^{2} p}{(2 \pi)^{2}} b_{l}^{\dagger}(\mathbf{p}) b_{l^{\prime}}(\mathbf{p}-\mathbf{k}) e^{-(i / 4 \pi) k_{x}\left(2 p_{y}-k_{y}\right)} \\
& +\int_{\frac{d^{2} k d^{2} k^{\prime}}{(2 \pi)^{4}} \sum_{l, l^{\prime}} \frac{e^{2} \omega_{c}}{4 \pi} \boldsymbol{a}\left(\tilde{\mathbf{k}^{\prime}}\right) \cdot \boldsymbol{a}(\tilde{\mathbf{k}}) f_{l, l^{\prime}}^{0}\left(\tilde{\mathbf{k}}+\tilde{\mathbf{k}}^{\prime}\right)} \\
& \times \int_{\mathrm{BZ}} \frac{d^{2} p}{(2 \pi)^{2}} b_{l}^{\dagger}(\mathbf{p}) b_{l^{\prime}}\left(\mathbf{p}-\mathbf{k}-\mathbf{k}^{\prime}\right) e^{-(i / 4 \pi)\left(k_{x}\right.} \\
& \left.+k_{x}^{\prime}\right)\left(2 p_{y}-k_{y}-k_{y}^{\prime}\right)+\sum_{l} \int \frac{d^{2} k}{(2 \pi)^{2}} v_{l}^{\mathrm{HF}}(\mathbf{k})\left\langle\bar{\rho}_{l}(-\tilde{\mathbf{k}})\right\rangle \bar{\rho}_{l}(\tilde{\mathbf{k}}),
\end{aligned}
$$

where $f_{l_{1}, l_{2}}^{\mu}(\mathbf{k})$ is defined by (see Appendix C)

$$
f_{l_{1}, l_{2}}^{\mu}(\mathbf{k})=\left\langle f_{l_{1}}\left|\frac{1}{2}\left\{v^{\mu}, e^{i \mathbf{k} \cdot \xi}\right\}\right| f_{l_{2}}\right\rangle,
$$

in which $v^{\mu}=\left(1,-\omega_{c} \eta, \omega_{c} \xi\right)$ is the electron velocity. Repeated Greek indices $\mu$ and $\nu$ are summed in this paper. The action is given by

$$
S\left[a, b, b^{\dagger}\right]=\int d t\left[\int_{\mathrm{BZ}} \frac{d^{2} p}{(2 \pi)^{2}} b_{l}^{\dagger}(\mathbf{p}, t)\left(i \partial_{t}+\mu_{F}\right) b_{l}(\mathbf{p}, t)-H(t)\right],
$$

where $H(t)$ is the Heisenberg representation of $H$.
Let us concentrate on the striped Hall state at half filling. Substituting Eq. (2.17) into Eq. (3.4), the action of the striped Hall state is given by

$$
\begin{aligned}
S_{\mathrm{HF}}\left[a, b, b^{\dagger}\right]= & \sum_{l, l^{\prime}} \int_{\mathrm{BZ}} \frac{d^{3} p d^{3} p^{\prime}}{(2 \pi)^{6}} b_{l}^{\dagger}(p)\left\{\left[p_{0}-\xi_{l}(\mathbf{p})\right]\right. \\
& \times \delta_{l, l^{\prime}}(2 \pi)^{3} \delta^{3}\left(p-p^{\prime}\right) \\
& \left.-U_{a_{1}}^{\left(l, l^{\prime}\right)}\left(p, p^{\prime}\right)-U_{a_{2}}^{\left(l, l^{\prime}\right)}\left(p, p^{\prime}\right)\right\} b_{l^{\prime}}\left(p^{\prime}\right),
\end{aligned}
$$

where

$$
\begin{aligned}
& U_{a_{1}}^{\left(l, l^{\prime}\right)}\left(p, p^{\prime}\right)=-\sum_{\mathbf{N}} e f_{l, l^{\prime}}^{\mu}\left(\widetilde{\mathbf{p}}-\widetilde{\mathbf{p}}^{\prime}-2 \pi \widetilde{\mathbf{N}}\right) h\left(\mathbf{p}+\mathbf{p}^{\prime}, \mathbf{N}\right) a_{\mu}\left(\widetilde{\mathbf{p}}-\widetilde{\mathbf{p}}^{\prime}\right. \\
&\left.-2 \pi \widetilde{\mathbf{N}}, p_{0}-p_{0}^{\prime}\right) e^{-(i / 4 \pi)\left(p_{x}-p_{x}^{\prime}\right)\left(p_{y}+p_{y}^{\prime}\right)} \\
& U_{a_{2}}^{\left(l, l^{\prime}\right)}\left(p, p^{\prime}\right)= \sum_{\mathbf{N}} \int \frac{d^{3} k}{(2 \pi)^{3}} \frac{e^{2} \omega_{c}}{4 \pi} f_{l, l^{\prime}}^{0}\left(\widetilde{\mathbf{p}}-\widetilde{\mathbf{p}}^{\prime}-2 \pi \widetilde{\mathbf{N}}\right) h(\mathbf{p} \\
&\left.+\mathbf{p}^{\prime}, \mathbf{N}\right) \boldsymbol{a}\left(\tilde{\mathbf{k}}, k_{0}\right) \cdot \boldsymbol{a}\left(\widetilde{\mathbf{p}}-\widetilde{\mathbf{p}}^{\prime}-\tilde{\mathbf{k}}-2 \pi \widetilde{\mathbf{N}}, p_{0}-p_{0}^{\prime}\right. \\
&\left.-k_{0}\right) e^{-(i / 4 \pi)\left(p_{x}-p_{x}^{\prime}\right)\left(p_{y}+p_{y}^{\prime}\right)}, \\
& h(\mathbf{p}, \mathbf{N}) \equiv(-1)^{N_{x}+N_{y}+N_{x} N_{y}} e^{-(i / 2) p_{x} N_{y}+(i / 2) p_{y} N_{x}} .
\end{aligned}
$$

Here, $p$ denotes $\left(\mathbf{p}, p_{0}\right), \xi_{l}(\mathbf{p})=E_{l}+\epsilon_{l}(\mathbf{p})-\mu_{F}$, and $U_{a_{1}}$ and $U_{a_{2}}$ are the first order term and the second order term with respect to $a_{\mu}$, respectively.

The partition function $Z[a]$ is calculated using path integrals by

$$
\begin{aligned}
Z[a] & =\int \mathcal{D} b^{\dagger} \mathcal{D} b e^{i S_{\mathrm{HF}}\left[a, b, b^{\dagger}\right]}=\int \mathcal{D} b^{\dagger} \mathcal{D} b e^{-(-i) \Sigma b^{\dagger}\left(g^{-1} \mathbf{1}-U_{a_{1}}-U_{a_{2}}\right) b} \\
& =e^{\operatorname{Tr} \log \left[(-i) g^{-1}\right]} e^{\operatorname{Tr} \log \left(\mathbf{1}-g U_{a_{1}}-g U_{a_{2}}\right)},
\end{aligned}
$$

where the power of the exponent is expressed in the matrix representation in the momentum space and $\operatorname{Tr}$ denotes the trace of the momentum indices and the LL indices. $g_{l}$ is the Green's function given by

$$
g_{l}(p)=\frac{\theta\left[\xi_{l}(\mathbf{p})\right]}{p_{0}-\xi_{l}(\mathbf{p})+i \delta}+\frac{\theta\left[-\xi_{l}(\mathbf{p})\right]}{p_{0}-\xi_{l}(\mathbf{p})-i \delta},
$$

where $\delta$ is an infinitesimal positive constant. The effective action $S_{\text {eff }}$ is defined as $S_{\text {eff }}[a]=-i \log Z[a]$. It consists of the nonperturbed part $S_{0}=-i \operatorname{Tr} \log \left[(-i) g^{-1}\right]$ and the correction part due to the external gauge field $\Delta S_{\text {eff }}[a] . \Delta S_{\text {eff }}[a]$ is given by (Fig. 7),

$$
\Delta S_{\mathrm{eff}}[a]=\Delta S_{1}[a]+\Delta S_{2}[a]+\Delta S_{3}[a]+\mathcal{O}\left[a^{3}\right]
$$

where

$$
\begin{aligned}
& \Delta S_{1}[a]=i \operatorname{Tr}\left[g U_{a_{1}}\right]=i \sum_{l} \int_{\mathrm{BZ}} \frac{d^{3} p}{(2 \pi)^{3}} g_{l}(p) U_{a_{1}}^{(l, l)}(p, p), \\
& \Delta S_{2}[a]=i \operatorname{Tr}\left[g U_{a_{2}}\right]=i \sum_{l} \int_{\mathrm{BZ}} \frac{d^{3} p}{(2 \pi)^{3}} g_{l}(p) U_{a_{2}}^{(l, l)}(p, p),
\end{aligned}
$$


(a)

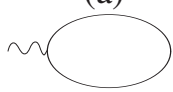

\section{(c)}

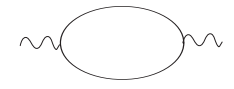

FIG. 7. (a), (b), and (c) are Feynman diagrams for $\Delta S_{1}, \Delta S_{2}$, and $\Delta S_{3}$, respectively.

$$
\begin{aligned}
\Delta S_{3}[a]= & \frac{i}{2} \operatorname{Tr}\left[g U_{a_{1}} g U_{a_{1}}\right]=\frac{i}{2} \sum_{l, l^{\prime}} \int_{\mathrm{BZ}} \frac{d^{3} p d^{3} p^{\prime}}{(2 \pi)^{6}} g_{l}(p) \\
& \times U_{a_{1}}^{\left(l, l^{\prime}\right)}\left(p, p^{\prime}\right) g_{l^{\prime}}\left(p^{\prime}\right) U_{a_{1}}^{\left(l^{\prime}, l\right)}\left(p^{\prime}, p\right) .
\end{aligned}
$$

Substituting the expressions for $g, U_{a 1}$, and $U_{a 2}$ into Eq. (3.10), $\Delta S_{\text {eff }}[a]$ is given by

$$
\begin{aligned}
\Delta S_{\mathrm{eff}}[a]= & \left(e l_{0}\right) a_{0}(0)+\sum_{N_{x}} e f_{l_{0}, l_{0}}^{\mu}\left(-2 \pi \tilde{N}_{x}, 0\right) \frac{\sin \left(p_{F} N_{x}\right)}{\pi N_{x}} \\
& \times e^{i \pi N_{x}} a_{\mu}\left(-2 \pi \tilde{N}_{x}, 0\right)-\frac{1}{2} \int \frac{d^{3} p}{(2 \pi)^{3}} \sum_{\mathbf{N}} a_{\mu}\left(\mathbf{p}, p_{0}\right) \\
& \times K^{\mu \nu}(p, \mathbf{N}) a_{\nu}\left(-\mathbf{p}-2 \pi \tilde{\mathbf{N}},-p_{0}\right)
\end{aligned}
$$

where $l_{0}$ represents the uppermost partially filled LL. $K^{\mu \nu}(p, \mathbf{N})$ is a response function given by

$$
\begin{aligned}
K^{\mu \nu}(p, \mathbf{N})= & \sum_{l, l^{\prime}} e^{2} f_{l, l^{\prime}}^{\mu}(\mathbf{p}) f_{l^{\prime}, l}^{\nu}(-\mathbf{p}-2 \pi \tilde{\mathbf{N}}) I_{l, l^{\prime}}\left(p_{0}, \hat{\mathbf{p}}, \mathbf{N}\right) h(\hat{\mathbf{p}}, \mathbf{N}) \\
& +\frac{e^{2}}{2 \pi} \omega_{c} \sum_{\mathbf{N}}\left[l_{0} \delta_{\mathbf{N}, 0}+f_{l_{0}, l_{0}}^{0}(-2 \pi \tilde{\mathbf{N}})\right. \\
& \left.\times(-1)^{N_{x}} \frac{\sin \left(\pi N_{x} / 2\right)}{\pi N_{x}} \delta_{N_{y}, 0}\right]\left(\delta_{\mu, 1} \delta_{\nu, 1}+\delta_{\mu, 2} \delta_{\nu, 2}\right),
\end{aligned}
$$

where $I_{l, l^{\prime}}\left(p_{0}, \mathbf{p}, \mathbf{N}\right)$ is the loop integral in Fig. 7(c), which is given by

$$
\begin{aligned}
I_{l, l^{\prime}}\left(p_{0}, \mathbf{p}, \mathbf{N}\right)= & \int_{\mathrm{BZ}} \frac{d^{2} p^{\prime}}{(2 \pi)^{2}}\left\{\frac{\theta\left[\xi_{l}\left(\mathbf{p}+\mathbf{p}^{\prime}\right)\right] \theta\left[-\xi_{l^{\prime}}\left(\mathbf{p}^{\prime}\right)\right]}{p_{0}+\xi_{l^{\prime}}\left(\mathbf{p}^{\prime}\right)-\xi_{l}\left(\mathbf{p}+\mathbf{p}^{\prime}\right)+i \delta}\right. \\
& \left.-\frac{\theta\left[-\xi_{l}\left(\mathbf{p}+\mathbf{p}^{\prime}\right)\right] \theta\left[\xi_{l^{\prime}}\left(\mathbf{p}^{\prime}\right)\right]}{p_{0}+\xi_{l^{\prime}}\left(\mathbf{p}^{\prime}\right)-\xi_{l}\left(\mathbf{p}+\mathbf{p}^{\prime}\right)-i \delta}\right\} e^{-i p_{x}^{\prime} N_{y}+i p_{y}^{\prime} N_{x}},
\end{aligned}
$$

in which the $p_{0}^{\prime}$ integral has been performed. In Eq. (3.12), the first term and the second term come from $\Delta S_{3}[a]$ and $\Delta S_{2}[a]$, respectively, and the second term is cancelled with the $p=0$ part of the first term, as expected from gauge invariance. Hence, $K^{i, i}(p=0, \mathbf{N})=0$ for $i=1,2$.

In the long wavelength limit, the largest contribution in the response function comes from the $\mathbf{N}=0$ part. In the case of $p_{0}=p_{y}=0$ and $p_{x} \rightarrow 0$, which is used in the next section, the largest contribution comes from the lowest order term in $K_{0}^{\mu \nu}\left(p_{x}\right) \equiv K^{\mu \nu}\left(p_{x}, 0\right)$ with respect to $p_{x}$. By expanding $K_{0}^{\mu \nu}\left(p_{x}\right)$ up to the lowest order, the response functions in the long wavelength limit are given as

$$
\begin{aligned}
& K_{0}^{00}\left(p_{x}\right)=-\frac{\sigma_{x y}^{(\nu)}}{\omega_{c}} p_{x}^{2}, \\
& K_{0}^{0 y}\left(p_{x}\right)=-i \sigma_{x y}^{(\nu)} p_{x}, \\
& K_{0}^{y 0}\left(p_{x}\right)=i \sigma_{x y}^{(\nu)} p_{x}, \\
& K_{0}^{y y}\left(p_{x}\right)=\alpha_{K} \omega_{c} p_{x}^{2},
\end{aligned}
$$

where $\sigma_{x y}^{(\nu)}=e^{2} \nu / 2 \pi$ and $\alpha_{K}=e^{2} \omega_{c}\left(l_{0}^{2}+2 l_{0} \nu^{*}+\nu^{*}\right) / 4 \pi^{2} . \sigma_{x y}^{(\nu)}$ is identified as the Hall conductance since if we consider a static homogeneous electric field in the $x$ direction generated by the gauge field $a_{0}^{\mathrm{ex}}(x)=x E_{x}$, then the electric current in the $y$ direction $j^{y}(x)$ is given in the long wavelength limit by $\left\langle j_{y}(x)\right\rangle=\delta \Delta S_{\mathrm{eff}} / \delta a_{y}(x)=K_{0}^{y 0}\left(\partial_{x}\right) a_{0}(x)=-\sigma_{x y}^{(\nu)} E_{x}$, where the response function transformed in the coordinate space is used. The longitudinal resistivity becomes zero in the present calculation since the impurity potential is not included. If impurities are added, it is expected that the longitudinal resistivity becomes zero in one direction and finite in the other direction due to the anisotropic Fermi surface.

\section{B. Response function of the anisotropic charge density wave state}

The action of the ACDW state at half-filling is given by

$$
S_{\mathrm{HF}}\left[a, \mathbf{c}, \mathbf{c}^{\dagger}\right]=\sum_{l, l^{\prime}} \int_{\mathrm{RBZ}} \frac{d^{3} p d^{3} p^{\prime}}{(2 \pi)^{6}} \mathbf{c}_{l}^{\dagger}(p)\left[G_{l}^{-1}(p) \delta_{l, l^{\prime}}(2 \pi)^{3} \delta^{3}\left(p-p^{\prime}\right)-V_{a_{1}}^{\left(l, l^{\prime}\right)}\left(p ; p^{\prime}\right)-V_{a_{2}}^{\left(l, l^{\prime}\right)}\left(p ; p^{\prime}\right)\right] \mathbf{c}_{l^{\prime}}\left(p^{\prime}\right),
$$

where $V_{a_{j}}(j=1,2)$ is a $2 \times 2$ matrix given by

$$
\begin{gathered}
V_{a_{j}}^{\left(l, l^{\prime}\right)}\left(p, p^{\prime}\right)=U^{\dagger}(p) \mathbf{U}_{a_{j}}^{\left(l, l^{\prime}\right)}\left(p, p^{\prime}\right) U(p), \\
\mathbf{U}_{a_{j}}^{\left(l, l^{\prime}\right)}\left(p ; p^{\prime}\right)=\left(\begin{array}{cc}
U_{a_{j}}^{\left(l, l^{\prime}\right)}\left(p ; p^{\prime}\right) & U_{a_{j}}^{\left(l, l^{\prime}\right)}\left(p ; p_{x}^{\prime}, p_{y}^{\prime}+\pi, p_{0}^{\prime}\right) \\
U_{a_{j}}^{\left(l, l^{\prime}\right)}\left(p_{x}, p_{y}+\pi, p_{0} ; p^{\prime}\right) & U_{a_{j}}^{\left(l, l^{\prime}\right)}\left(p_{x}, p_{y}+\pi, p_{0} ; p_{x}^{\prime}, p_{y}^{\prime}+\pi, p_{0}^{\prime}\right)
\end{array}\right),
\end{gathered}
$$


and $G_{l}$ is a $2 \times 2$ matrix Green's function given by

$$
G_{l}(p)=\left\{\begin{array}{cc}
\left(\begin{array}{cc}
g_{l}^{+}(p) & 0 \\
0 & g_{l}^{-}(p)
\end{array}\right) & \text { for } l=l_{0} \\
g_{l}^{-}(p) \mathbf{1} & \text { for } l<l_{0} \\
g_{l}^{+}(p) \mathbf{1} & \text { for } l>l_{0}
\end{array} .\right.
$$

In Eq. (3.17), $g_{l}^{ \pm}(p)=1 /\left[p_{0}-\xi_{l}^{ \pm}(p) \pm i \delta\right]$ is a one-particle Green's function for the upper or lower band, $\mathbf{1}$ is a $2 \times 2$ unit matrix, and $\xi_{l}^{ \pm}(\mathbf{p})=E_{l_{0}}+\epsilon_{ \pm}^{\left(l_{0}\right)}(\mathbf{p})-\mu_{F}$.

The partition function $Z[a]$ is calculated using path integrals by

$$
Z[a]=\int \mathcal{D} \mathbf{c}^{\dagger} \mathcal{D} \mathbf{c} e^{i S_{\mathrm{HF}}\left[a, \mathbf{c}, c^{\dagger}\right]}=e^{\operatorname{Tr} \log \left[(-i) G^{-1}\right]} e^{\operatorname{Tr} \log \left(\mathbf{1}-G V_{a_{1}}-G V_{a_{2}}\right)} .
$$

The correction part of the effective action $\Delta S_{\text {eff }}[a]$ is given by

$$
\Delta S_{\mathrm{eff}}[a]=\Delta S_{1}[a]+\Delta S_{2}[a]+\Delta S_{3}[a]+\mathcal{O}\left[a^{3}\right],
$$

where

$$
\begin{aligned}
\Delta S_{1}[a]= & i \operatorname{Tr}\left[G V_{a_{1}}\right]=i \sum_{l} \int_{\mathrm{RBZ}} \frac{d^{3} p}{(2 \pi)^{3}} \operatorname{Tr}_{2 \times 2} \\
& \times\left[G_{l}(p) V_{a_{1}}^{(l, l)}(p, p)\right],
\end{aligned}
$$

$$
\begin{gathered}
\Delta S_{2}[a]=i \operatorname{Tr}\left[G V_{a_{2}}\right]=i \sum_{l} \int_{\mathrm{RBZ}} \frac{d^{3} p}{(2 \pi)^{3}} \operatorname{Tr}_{2 \times 2} \\
\times\left[G_{l}(p) V_{a_{2}}^{(l, l)}(p, p)\right], \\
\Delta S_{3}[a]=\frac{i}{2} \operatorname{Tr}\left[G V_{a_{1}} G V_{a_{1}}\right]=\frac{i}{2} \sum_{l, l^{\prime}} \int_{\mathrm{RBZ}} \frac{d^{3} p d^{3} p^{\prime}}{(2 \pi)^{6}} \operatorname{Tr}_{2 \times 2}\left[G_{l}(p)\right. \\
\left.\quad \times V_{a_{1}}^{\left(l, l^{\prime}\right)}\left(p, p^{\prime}\right) G_{l^{\prime}}\left(p^{\prime}\right) V_{a_{1}}^{\left(l^{\prime}, l\right)}\left(p^{\prime}, p\right)\right] .
\end{gathered}
$$

Here, $\operatorname{Tr}$ denotes the trace with respect to the momentum indices, the LL indices, and the $2 \times 2$ matrix indices, and $\operatorname{Tr}_{2 \times 2}$ denotes the trace with respect to only the $2 \times 2$ matrix indices. Substituting the expressions for $G, V_{a_{1}}, V_{a_{2}}$ into Eq. (3.20), we obtain the following expression for the $\mathbf{N}=0$ part of $\Delta S_{\text {eff }}$ as in the case of the striped Hall state:

$$
\begin{aligned}
\Delta S_{\mathrm{eff}}[a]= & e\left(l_{0}+\frac{1}{2}\right) a_{0}(0)-\frac{1}{2} \int \frac{d^{3} p}{(2 \pi)^{3}} a_{\mu}\left(\mathbf{p}, p_{0}\right) \\
& \times K_{0}^{\mu \nu}(p) a_{\nu}\left(-\mathbf{p},-p_{0}\right),
\end{aligned}
$$

where $K_{0}^{\mu \nu}$ is given by

$$
\begin{aligned}
K_{0}^{\mu \nu}(p)= & -\sum_{l<l_{0}} \sum_{l^{\prime}>l_{0}} \frac{e^{2}}{\omega_{c}\left(l^{\prime}-l\right)}\left[f_{l, l^{\prime}}^{\mu}(\mathbf{p}) f_{l^{\prime}, l}^{\nu}(-\mathbf{p})+f_{l^{\prime}, l}^{\mu}(\mathbf{p}) f_{l, l^{\prime}}^{\nu}(-\mathbf{p})\right]+\frac{1}{2}\left(-\sum_{l<l_{0}}+\sum_{l>l_{0}}\right) \frac{e^{2}}{\omega_{c}\left(l_{0}-l\right)}\left[f_{l, l_{0}}^{\mu}(\mathbf{p}) f_{l_{0}, l}^{\nu}(-\mathbf{p})+f_{l_{0}, l}^{\mu}(\mathbf{p}) f_{l, l_{0}}^{\nu}(-\mathbf{p})\right] \\
& +\int_{\operatorname{RBZ}} \frac{d^{2} p^{\prime}}{(2 \pi)^{2}} \frac{1}{p_{0}-\left[\epsilon\left(\mathbf{p}^{\prime}\right)+\epsilon\left(\hat{\mathbf{p}}+\mathbf{p}^{\prime}\right)\right]}\left[1-\frac{A\left(\hat{\mathbf{p}}+\mathbf{p}^{\prime}\right) A\left(\mathbf{p}^{\prime}\right)+\operatorname{Re}\left(B\left(\hat{\mathbf{p}}+\mathbf{p}^{\prime}\right) B^{*}\left(\mathbf{p}^{\prime}\right) e^{\left.-(i / 2) \hat{p}_{x}\right)}\right.}{\epsilon\left(\hat{\mathbf{p}}+\mathbf{p}^{\prime}\right) \boldsymbol{\epsilon}\left(\mathbf{p}^{\prime}\right)}\right] e^{2} f_{l_{0}, l_{0}}^{\mu}(\mathbf{p}) f_{l_{0}, l_{0}}^{\nu}(-\mathbf{p}) \\
& +\left(l_{0}+\frac{1}{2}\right) \frac{e^{2}}{2 \pi} \omega_{c}\left(\delta_{\mu, 1} \delta_{\nu, 1}+\delta_{\mu, 2} \delta_{\nu, 2}\right),
\end{aligned}
$$

up to $\mathcal{O}\left[\epsilon(\mathbf{p}) / \omega_{C}\right]$. In Eq. (3.22), the last term is cancelled with the $p=0$ term of the second term, as expected from gauge invariance again. Hence, $K_{0}^{i, i}(0)=0$ for $i=1,2$.

In the long wavelength limit $p_{0}=p_{y}=0$ and $p_{x} \rightarrow 0$, the largest contribution in the response function comes from the lowest order term with respect to $p_{x}$. The expressions of $K_{0}^{0 y}$ and $K_{0}^{y 0}$ become the same as in the case of the striped Hall state. The expression of $K_{0}^{00}$ becomes slightly different by the correction from the intra-LL effect at the uppermost partially filled LL. For the striped Hall state, the one-particle energy shown in Fig. 3 has the inter-LL energy gap in the $p_{x}$ direction, and the inter-LL effect gives the response functions given in Eq. (3.12). For the ACDW state, the one-particle energy shown in Fig. 6 has the intra-LL energy gap in the $p_{x}$ direction as well as the inter-LL energy gap. While the inter-LL effect gives the same expression of the response function as that of the striped Hall state, the intra-LL effect causes some corrections to the response function. Including these corrections, $K_{0}^{00}$ of the ACDW state in the long wavelength limit is given by (see Appendix D)

$$
K_{0}^{00}\left(p_{x}\right)=-\left(1+\frac{\sqrt{B}}{\nu} \beta\right) \frac{\sigma_{x y}^{(\nu)}}{\omega_{c}} p_{x}^{2},
$$

where $a=\sqrt{2 \pi / e B}$ is used explicitly in order to compare the theoretical results with experimental data. The value of $\beta$ at each LL is shown in Table III. Note that the unit of $\beta$ is (tesla $)^{-1 / 2}$. The Hall conductance is given by $\sigma_{x y}^{(\nu)}$, as in the case of the striped Hall state. The longitudinal resistivity becomes zero in the present calculation since the impurity potential is not included. However, it is expected that the longitudinal resistivity remains zero even in the system with impurities because of the energy gaps. 
TABLE III. The value of $\beta$ at each LL. "Parallel" is the case in which the ACDW direction faces the $y$ direction. "Perpendicular" is the case in which the ACDW direction faces the $x$ direction. The unit of $\beta$ is $\mathrm{T}^{-1 / 2}$.

\begin{tabular}{lcc}
\hline \hline$l$ & Parallel & Perpendicular \\
\hline 0 & 0.497 & 0.497 \\
1 & 0.386 & 0.824 \\
2 & 0.472 & 1.044 \\
3 & 0.581 & 1.171 \\
\hline \hline
\end{tabular}

\section{ENERGY CORRECTIONS DUE TO FINITE ELECTRIC CURRENTS}

In this section, we consider the quantum Hall system with an injected electric current and investigate the current effect on the striped Hall state and the ACDW state. Effects of impurities and metallic contacts are ignored in our calculations. For the striped Hall state, we only consider the current parallel to the stripe direction since in this case, the current effect can be estimated with no ambiguity even in the system with impurities. When the current flows in the stripe direction, charges accumulate around both edges of the sample in the perpendicular direction, as we will see later, and the electric field generates in the perpendicular direction. In this case, the impurity effect is negligible since the Fermi surface has the inter-LL energy gap in the perpendicular direction. On the other hand, when the current flows in the perpendicular direction, the impurity effect becomes relevant since the electric field generates in the stripe direction while the Fermi surface is gapless in this direction. The current effect in this case is nontrivial and will be studied in future work.

In the system with an injected current, it is naively expected that the current flow causes the plus and minus charge accumulation at both edges of the sample with the opposite sign, as expected from the classical Hall effect. MacDonald et al. have studied the injected current effect on the integer quantum Hall system about two decades ago. ${ }^{23}$ They have calculated the current and charge distributions and found that the charge accumulation occurs in the integer quantum Hall system. The charge accumulation causes the energy correction via the Coulomb interaction between the accumulated charges. It is expected that the same type of the energy correction exists even in the present highly correlated quantum Hall states. However, it has not been studied as far as the present authors know. In the following, we first derive the current and charge distributions in the striped Hall state and the ACDW state using the effective action. Then, we estimate the current dependence of the energy corrections of the two HF states. It is shown that the energy of the ACDW state increases faster than that of the striped Hall state as the injected current increases.

\section{A. Current and charge distributions}

We study current and charge distributions of the striped Hall state and the ACDW state. We denote the two-point function in the HF theory with no injected current
$\left\langle\Psi^{\dagger}(\mathbf{x}, t) \Psi\left(\mathbf{x}^{\prime}, t\right)\right\rangle_{I=0}$ as $F\left(\mathbf{x}, \mathbf{x}^{\prime}\right)$ for both states. In the system with a finite electric current, electromagnetic fields and the two-point function deviate from their original values. These deviations are taken into account in the calculation of the total energy. We define these deviations by

$$
\begin{gathered}
\boldsymbol{a}(\mathbf{x}, t)=\mathbf{A}(\mathbf{x}, t)-\mathbf{A}_{\mathrm{ex}}(\mathbf{x}), \\
\delta \rho\left(\mathbf{x}, \mathbf{x}^{\prime}, t\right)=\left\langle\Psi^{\dagger}(\mathbf{x}, t) \Psi\left(\mathbf{x}^{\prime}, t\right)\right\rangle-F\left(\mathbf{x}, \mathbf{x}^{\prime}\right),
\end{gathered}
$$

where $\boldsymbol{a}$ and $\delta \rho$ are unspecified for the moment and will be determined later. The total action in the Coulomb gauge $\nabla \cdot \mathbf{A}(x)=0$ is given as

$$
\begin{aligned}
S_{\mathrm{tot}}\left[\mathbf{A}, \Psi^{\dagger}, \Psi\right]= & \int d t d^{3} x\left\{\frac{\boldsymbol{\epsilon}}{2} \dot{\mathbf{A}}^{2}(\mathbf{x}, t)-\frac{1}{2 \mu}[\nabla \times \mathbf{A}(\mathbf{x}, t)]^{2}\right\} \\
& +\int d t d^{3} x \Psi^{\dagger}(\mathbf{x}, t)\left\{i \partial_{t}-\frac{[\mathbf{p}+e \mathbf{A}(\mathbf{x}, t)]^{2}}{2 m}\right\} \\
& \times \Psi(\mathbf{x}, t) \delta(z) \\
& -\frac{1}{2} \int d t d^{3} x d^{3} x^{\prime} \Psi^{\dagger}(\mathbf{x}, t) \Psi^{\dagger}\left(\mathbf{x}^{\prime}, t\right) V(\mathbf{x} \\
& \left.-\mathbf{x}^{\prime}\right) \Psi\left(\mathbf{x}^{\prime}, t\right) \Psi(\mathbf{x}, t) \delta(z) \delta\left(z^{\prime}\right),
\end{aligned}
$$

where $\mu$ is the magnetic constant and the dot means the time derivative. This total action consists of the three-dimensional electromagnetic field term and the two-dimensional electron field term. In the Coulomb gauge, the interaction between electric fields is expressed by the Coulomb interaction. Applying the HF approximation to the Coulomb interaction part and substituting Eq. (4.1), the total action is rewritten by

$$
S_{\mathrm{tot}}\left[\boldsymbol{a}, \delta \rho, \Psi^{\dagger}, \Psi\right]=S_{\mathrm{EM}}[\boldsymbol{a}]+S_{\mathrm{HF}}\left[\boldsymbol{a}, \delta \rho, \Psi^{\dagger}, \Psi\right],
$$

where

$$
\begin{aligned}
S_{\mathrm{EM}}[\boldsymbol{a}]=\int d t & d^{3} x\left\{\frac{\boldsymbol{\epsilon}}{2} \dot{\boldsymbol{a}}^{2}(\mathbf{x}, t)-\frac{1}{2 \mu}[\nabla \times \boldsymbol{a}(\mathbf{x}, t)]^{2}\right\}, \\
S_{\mathrm{HF}}\left[\boldsymbol{a}, \delta \rho, \Psi^{\dagger}, \Psi\right]= & \int d t d^{3} x \Psi^{\dagger}(\mathbf{x}, t)\left\{i \partial_{t}\right. \\
& \left.-\frac{\left[\mathbf{p}+e \mathbf{A}_{\mathrm{ex}}(\mathbf{x})+e \boldsymbol{a}(\mathbf{x}, t)\right]^{2}}{2 m}\right\} \Psi(\mathbf{x}, t) \delta(z) \\
& -\int d t d^{3} x d^{3} x^{\prime}\{[F(\mathbf{x}, \mathbf{x})+\delta \rho(\mathbf{x}, \mathbf{x}, t)] \\
& \times V\left(\mathbf{x}-\mathbf{x}^{\prime}\right) \Psi^{\dagger}\left(\mathbf{x}^{\prime}, t\right) \Psi\left(\mathbf{x}^{\prime}, t\right)-\left[F\left(\mathbf{x}, \mathbf{x}^{\prime}\right)\right. \\
& \left.+\delta \rho\left(\mathbf{x}, \mathbf{x}^{\prime}, t\right)\right] V\left(\mathbf{x}-\mathbf{x}^{\prime}\right) \Psi^{\dagger}\left(\mathbf{x}^{\prime}, t\right) \\
& \times \Psi(\mathbf{x}, t)\} \delta(z) \delta\left(z^{\prime}\right) .
\end{aligned}
$$

In the expression of $S_{\mathrm{EM}}$, the term of the uniform external magnetic field is dropped since it gives only the same energy constant to the two HF states. In Eq. (4.4), the term including $\delta \rho(\mathbf{x}, \mathbf{x}, t)$ and the term including $\delta \rho\left(\mathbf{x}, \mathbf{x}^{\prime}, t\right)$ are the Hartree term and the Fock term, respectively. As seen in Appendix A, the Fock term becomes negligible compared to the Hartree term in the long wavelength limit since in the momentum space, the Hartree term is proportional to the Coulomb po- 
tential $V(\mathbf{k})$, which is $\mathcal{O}(1 / k)$, and gives a larger contribution than the Fock term for the small momentum $k$. In the following calculation, the deviation of the Fock term is dropped. If we introduce the potential generated by the electron density deviation as

$$
a_{0}(\mathbf{x}, t) \equiv \int d^{3} x^{\prime} \frac{(-e) \delta \rho\left(\mathbf{x}^{\prime}, \mathbf{x}^{\prime}, t\right)}{4 \pi \epsilon\left|\mathbf{x}-\mathbf{x}^{\prime}\right|} \delta\left(z^{\prime}\right)
$$

$S_{\mathrm{HF}}$ is rewritten as

$$
\begin{aligned}
S_{\mathrm{HF}}\left[\boldsymbol{a}, a_{0}, \Psi^{\dagger}, \Psi\right]= & \int d t d^{3} x \Psi^{\dagger}(\mathbf{x}, t)\left\{i \partial_{t}+e a_{0}(\mathbf{x}, t)\right. \\
& \left.-\frac{\left[\mathbf{p}+e \mathbf{A}_{\mathrm{ex}}(\mathbf{x})+e \boldsymbol{a}(\mathbf{x}, t)\right]^{2}}{2 m}\right\} \Psi(\mathbf{x}, t) \delta(z) \\
& -\int d t d^{3} x d^{3} x^{\prime}\left[F(\mathbf{x}, \mathbf{x}) V\left(\mathbf{x}-\mathbf{x}^{\prime}\right)\right. \\
& \times \Psi^{\dagger}\left(\mathbf{x}^{\prime}, t\right) \Psi\left(\mathbf{x}^{\prime}, t\right)-F\left(\mathbf{x}, \mathbf{x}^{\prime}\right) V\left(\mathbf{x}-\mathbf{x}^{\prime}\right) \\
& \left.\times \Psi^{\dagger}\left(\mathbf{x}^{\prime}, t\right) \Psi(\mathbf{x}, t)\right] \delta(z) \delta\left(z^{\prime}\right) .
\end{aligned}
$$

The same form of the action is obtained from the Hamiltonian in the system with the infinitesimal external gauge field shown in Eq. (3.1) when the Coulomb interaction part is approximated in the HF approximation. The important difference is that $a_{\mu}$ in the present case represents the finite gauge field induced by the current flow. Although the meaning of $a_{\mu}$ is different, the effective action obtained in the previous section is applicable as long as $a_{\mu}$ is small.

The partition function is given by

$$
Z=\int \mathcal{D} \boldsymbol{a} \int \mathcal{D} \Psi^{\dagger} \mathcal{D} \Psi e^{i S_{\mathrm{EM}}[\boldsymbol{a}]+i S_{\mathrm{HF}}\left[a, a_{0}, \Psi^{\dagger}, \Psi\right]}
$$

Integrating out electron fields and expanding the results up to second order of $\boldsymbol{a}$ and $a_{0}$, we obtain the effective action $S_{\text {eff }}$ as

$$
Z=\int \mathcal{D} \boldsymbol{a} e^{i S_{\mathrm{EM}}[\boldsymbol{a}]+i S_{0}+i \Delta S_{\mathrm{eff}}\left[\boldsymbol{a}, a_{0}\right]}
$$

The functional derivative of $\left(S_{\mathrm{EM}}+S_{0}+\Delta S_{\text {eff }}\right)$ with respect to $\boldsymbol{a}(\mathbf{x}, t)$ gives the Maxwell's equation for $\boldsymbol{a}(\mathbf{x}, t)$,

$$
\left(\boldsymbol{\epsilon} \partial_{t}^{2}-\frac{1}{\mu} \nabla^{2}\right) \boldsymbol{a}(\mathbf{x}, t)=\langle\mathbf{j}(\mathbf{x}, t)\rangle_{a} \delta(z),
$$

where $\mathbf{j}(\mathbf{x}, t)$ is a current operator and $\langle\hat{O}(x)\rangle_{a}$ means an expectation value of an operator $\hat{O}(x)$ for the system with finite $a_{\mu}$. The solution of this equation gives the stationary point of the action with respect to $a_{\mu}$. We use the action into which the solution of Eq. (4.9) is substituted as the effective action. $\langle\mathbf{j}(\mathbf{x}, t)\rangle_{a}$ and $\delta \rho(\mathbf{x}, t) \equiv \delta \rho(\mathbf{x}, \mathbf{x}, t)$ are calculated from the effective action by

$$
\frac{\delta \Delta S_{\mathrm{eff}}\left[\boldsymbol{a}, a_{0}\right]}{\delta \boldsymbol{a}(\mathbf{x}, t)}=\langle\mathbf{j}(\mathbf{x}, t)\rangle_{a} \delta(z),
$$

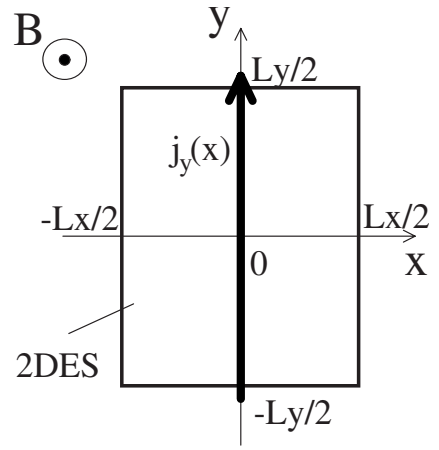

FIG. 8. Schematic view of the 2D electron system in a magnetic field with the injected current. The current flows in the $y$ direction and has only the $x$-coordinate dependence.

$$
-\frac{\delta \Delta S_{\mathrm{eff}}\left[\boldsymbol{a}, a_{0}\right]}{\delta a_{0}(\mathbf{x}, t)}=(-e)\left[\rho_{0}(\mathbf{x})+\delta \rho(\mathbf{x}, t)\right] \delta(z),
$$

where the $\rho_{0}(\mathbf{x})$ is the expectation value of the density operator in the system with no injected current. Equations (4.5), (4.9), and (4.10) determine $a_{0}(\mathbf{x}, t)$ and $\boldsymbol{a}(\mathbf{x}, t)$, or $\delta \rho(\mathbf{x}, t)$ and $\langle\mathbf{j}(\mathbf{x}, t)\rangle_{a}$, self-consistently.

We concentrate on the finite system with the static injected current flowing in the $y$ direction and depending only on $x$. The lengths of the 2D electron system in the $x$ direction and the $y$ direction are $L_{x}$ and $L_{y}$, respectively (Fig. 8). In this case, the electron density also depends only on $x$, and Eqs. (4.5) and (4.9) give the following solutions at $z=0$ :

$$
\begin{gathered}
a_{0}(x)=-\frac{1}{2 \pi \epsilon} \int_{-L_{x} / 2}^{L_{x} / 2} d x^{\prime} \ln \left|x-x^{\prime}\right|(-e) \delta \rho\left(x^{\prime}\right), \\
a_{y}(x)=\frac{\mu}{2 \pi} \int_{-L_{x} / 2}^{L_{x} / 2} d x^{\prime} \ln \left|x-x^{\prime}\right|\left\langle j_{y}\left(x^{\prime}\right)\right\rangle_{a} .
\end{gathered}
$$

As shown in the previous section, the effective action can be divided into the nonperturbed part and the correction part due to currents, and in the long wavelength limit, the correction part $\Delta S_{\text {eff }}$ is given as

$$
\begin{aligned}
\Delta S_{\mathrm{eff}}\left[\boldsymbol{a}, a_{0}\right]= & -T L_{y} \int_{-L_{x} / 2}^{L_{x} / 2} d x(-e) \bar{\rho}_{0} a_{0}(x) \\
& -\frac{T L_{y}}{2} \int_{-L_{x} / 2}^{L_{x} / 2} d x a_{\mu}(x) K_{0}^{\mu \nu}\left(\partial_{x}\right) a_{\nu}(x),
\end{aligned}
$$

where $\bar{\rho}_{0}$ is a uniform part of the density and $T$ is the total time. $K_{0}^{\mu \nu}\left(\partial_{x}\right)$ is the Fourier transformed form of the response function obtained in the previous section. Substituting Eq. (4.12) into Eq. (4.10), $\delta \rho(x)$ and $\left\langle j_{y}(x)\right\rangle_{a}$ are given as

$$
\begin{gathered}
(-e) \delta \rho(x)=K_{0}^{00}\left(\partial_{x}\right) a_{0}(x)-K_{0}^{0 y}\left(\partial_{x}\right) a_{y}(x), \\
\left\langle j_{y}(x)\right\rangle_{a}=K_{0}^{y 0}\left(\partial_{x}\right) a_{0}(x)-K_{0}^{y y}\left(\partial_{x}\right) a_{y}(x) .
\end{gathered}
$$

Equations (4.11) and (4.13) determine the current and charge density distributions up to an overall constant. The overall 


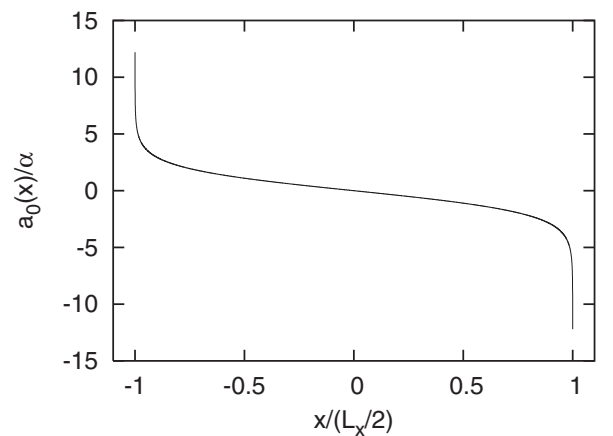

FIG. 9. Potential distribution $a_{0}(x)$. The first derivative of $a_{0}(x)$ gives the current distribution and the second derivative gives the charge distribution.

constant is determined by requiring the following constraints:

$$
\int_{-L_{x} / 2}^{L_{x} / 2} d x j_{y}(x)=I, \quad \int_{-L_{x} / 2}^{L_{x} / 2} d x \delta \rho(x)=0,
$$

where $I$ is a total current. Using the explicit form of the response functions derived in Sec. III, we obtain the integral equations to determine the current and charge distributions.

The same type of the integral equations has already been solved for the integer quantum Hall state. ${ }^{23-26}$ Their results are summarized as follows: (i) In Eq. (4.13), the terms including the vector potential $a_{y}(x)$ give a very small effect in the integral equations compared to the terms including the scalar potential $a_{0}(x)$, and the vector potential terms are negligible in a good approximation. (ii) The analytical solution of the integral equation without the vector potential term is obtained by means of the Wiener-Hopf technique. (iii) $a_{0}(x)=$ const $\times \ln \left|\left(x-L_{x} / 2\right) /\left(x+L_{x} / 2\right)\right|$ is the good approximate form of the analytical solution except near the edge, and the constant coefficient is determined from the constraint for the total current. The same results hold in our case.

The integral equation for the potential is given as

$$
a_{0}(x)=-\gamma \int_{-L_{x} / 2}^{L_{x} / 2} d x^{\prime} \ln \left|x-x^{\prime}\right| \partial_{x^{\prime}}^{2} a_{0}\left(x^{\prime}\right),
$$

where $\gamma=(1+\beta \sqrt{B} / \nu) \sigma_{x y}^{(\nu)} / 2 \pi \epsilon \omega_{c}$ ( $\beta=0$ for the striped Hall state). $\gamma$ has the dimension of length and is very small for the magnetic fields of the order of several tesla in the quantum Hall regime. For example, if $\epsilon=13 \epsilon_{0}, m=0.067 m_{e}$ (these are parameters in $\mathrm{GaAs}$ ), and $\beta=0$, then $\gamma$ is of the order of $10^{-8} \mathrm{~m}$. The current and charge distributions are obtained from $a_{0}(x)$ as

$$
(-e) \delta \rho(x)=2 \pi \epsilon \gamma \partial_{x}^{2} a_{0}(x), \quad\left\langle j_{y}(x)\right\rangle_{a}=-\sigma_{x y}^{(\nu)} \partial_{x} a_{0}(x) .
$$

The approximate solution of Eq. (4.15) is given by (Fig. 9)

$$
a_{0}(x)=\alpha \ln \left|\frac{x-L_{x} / 2}{x+L_{x} / 2}\right| \quad \text { for }|x| \leqslant \frac{L_{x}}{2}-\gamma,
$$

with a linear extrapolation of $a_{0}$ to $\pm I R_{H} / 2$ in the interval within $\gamma$ from the edge, where $\alpha=I R_{H} / 2\left[1+\ln \left(L_{x} / \gamma\right)\right]$ and
$R_{H}=1 / \sigma_{x y}^{(\nu)}$ is the Hall resistivity. One may verify that Eq. (4.17) is indeed the approximate solution of the integral equation [Eq. (4.15)] by substituting Eq. (4.17) into Eq. (4.15) and performing one partial integration.

\section{B. Energy corrections}

The energy correction due to the injected current per unit space-time volume is calculated from the effective action by $\left(S_{\mathrm{EM}}+\Delta S_{\mathrm{eff}}\right) / T L_{x} L_{y}$. Since in the present case of $\nu^{*}=1 / 2$, the area occupied by one particle at the uppermost partially filled LL is $2 a^{2}$ (here, the vNL constant $a$ is written explicitly), the energy correction per particle $\delta E$ is given by $\left[\left(S_{\mathrm{EM}}\right.\right.$ $\left.+\Delta S_{\text {eff }} / T L_{x} L_{y}\right] 2 a^{2}$. Substituting Eqs. (4.11) and (4.13) into this expression, the energy correction per particle is given by

$$
\delta E[I]=-\frac{e^{2}}{2 \pi \epsilon L_{x}} \int_{-L_{x} x^{\prime 2}}^{L_{x} / 2} d x d x^{\prime} \delta \rho(x) \ln \left|x-x^{\prime}\right| \delta \rho\left(x^{\prime}\right) .
$$

Substituting Eq. (4.16) into Eq. (4.18) and using Eq. (4.15), the energy correction is written by

$$
\delta E[I]=\frac{\left(\sigma_{x y}^{(\nu)}\right)^{2}}{2 \pi \epsilon \gamma L_{x} \omega_{c}^{2}} \int_{-L_{x} / 2}^{L_{x} / 2} d x a_{0}(x) \partial_{x}^{2} a_{0}(x) .
$$

The final result is obtained by substituting Eq. (4.17) into this expression and performing the $x$ integral,

$$
\delta E[I]=\frac{\pi \epsilon}{L_{x}\left(\sigma_{x y}^{(\nu)}\right)^{2}} \frac{\ln (2 / b)-1}{[\ln (2 / b)+1]^{2}} I^{2},
$$

where $b$ is a dimensionless constant given by $b=\gamma /\left(L_{x} / 2\right)$ $(\ll 1)$. This expression depends on the filling factor, the magnetic field strength, and experimental parameters. Since the actual filling factor includes the spin degree of freedom, we use $\nu_{\mathrm{ex}}=2 l_{0}+\nu^{*}$ for lower spin bands and $\nu_{\mathrm{ex}}=\left(2 l_{0}+1\right)+\nu^{*}$ for upper spin bands instead of $\nu$. The magnetic field strength is related to the filling factor by $B=h n_{e} / e \nu_{\mathrm{ex}}\left(n_{e}\right.$ is an electron density). For example, if $n_{e}=2.67 \times 10^{15} \mathrm{~m}^{-2}$, then the magnetic field strengths are $4.42 \mathrm{~T}\left(\nu_{\mathrm{ex}}=5 / 2\right), 3.15 \mathrm{~T}\left(\nu_{\mathrm{ex}}\right.$ $=7 / 2), 2.45 \mathrm{~T}\left(\nu_{\mathrm{ex}}=9 / 2\right), 2.01 \mathrm{~T}\left(\nu_{\mathrm{ex}}=11 / 2\right), 1.70 \mathrm{~T}\left(\nu_{\mathrm{ex}}\right.$ $=13 / 2)$, and so on. We use $\epsilon=13 \epsilon_{0}, m=0.067 m_{e}, n_{e}=2.67$ $\times 10^{15} \mathrm{~m}$, and $L_{x}=5 \times 10^{-3} \mathrm{~m}$ in order to estimate the values of energy corrections, which are the parameters used in the experiment by Lilly et al. ${ }^{6}$ Then, the energy correction is given by $\delta E[I]=C I^{2}\left(q^{2} / l_{B}\right)$, with the coefficient $C$ shown in Table IV.

As shown in Sec. II, in the system with no injected current, the energy of the ACDW state is slightly lower than that of the striped Hall state. The differences of energy per particle $\Delta E_{0}$ are $9.3 \times 10^{-3} \quad\left(l_{0}=1\right), 2.3 \times 10^{-3} \quad\left(l_{0}=2\right), \quad 1.4$ $\times 10^{-3}\left(l_{0}=3\right)$, and so on in units of $q^{2} / l_{B}$. When the finite current is injected, charges are accumulated in both edges with the opposite sign. The accumulated charges give the energy corrections $\delta E[I]$, which depend on the value of current $I$. Including these corrections, the energy difference between the striped Hall state and the ACDW state $\Delta E[I]$ $=-\Delta E_{0}+\left(\delta E_{\mathrm{ACDW}}[I]-\delta E_{\text {stripe }}[I]\right)$ varies depending on $I$. The current dependence of $\Delta E[I]$ is shown in Fig. 10. In Fig. 10, 
TABLE IV. Values of the coefficient $C$ in units of $\mathrm{nA}^{-2}$ and the critical current $I_{c}$ in units of nA. "Parallel" is the case in which the ACDW direction is parallel to the current. "Perpendicular" is the case in which the ACDW direction is perpendicular to the current.

\begin{tabular}{lcccc}
\hline \hline$\nu_{\text {ex }}$ & Stripe & Parallel & Perpendicular & $I_{c}$ \\
\hline $5 / 2$ & 325.0 & 330.6 & 335.6 & 0.041 \\
$7 / 2$ & 204.4 & 206.6 & 209.0 & 0.065 \\
$9 / 2$ & 144.7 & 146.1 & 147.6 & 0.040 \\
$11 / 2$ & 109.9 & 110.7 & 111.6 & 0.053 \\
$13 / 2$ & 87.44 & 88.08 & 88.68 & 0.047 \\
\hline \hline
\end{tabular}

only the parallel case is plotted for the ACDW states since it has a weaker current dependence than the perpendicular case does. The signs of the energy differences change at the critical values of current $I_{c}$. The critical values are shown in Table IV. The critical values are about $0.04-0.07 \mathrm{nA}$. The current used in the experiments ${ }^{6,7}$ is above $1 \mathrm{nA}$ and is much larger than the critical value. At $1 \mathrm{nA}$, the energy differences $\Delta \epsilon[I]$ become $5.6(\nu=5 / 2), 1.4 \quad(\nu=9 / 2)$, and $0.63(\nu$ $=13 / 2)$ in units of $q^{2} / l_{B}$, which are much larger than the original energy differences at a zero injected current. Hence, the striped Hall state becomes the lower energy state and should be realized in the experiments.

\section{SUMMARY}

In this paper, we have investigated the effect of the finite electric current on the striped Hall state and the ACDW state in the system with no impurities and no metallic contacts using the effective action. We calculated the electromagnetic response functions and obtained the effective action. For the striped Hall state, the current parallel to the stripe direction was investigated. In this case, the current effect can be estimated with no ambiguity even in the system with impurities. The current and charge distributions were determined for both states in the system with the injected current. It is found that the charge accumulation occurs around both edges with the opposite sign, just as in the case of the integer quantum Hall state studied by MacDonald et al. and other authors. ${ }^{23-26}$ We hope that current and charge distributions will be observed in experiments for anisotropic states. The

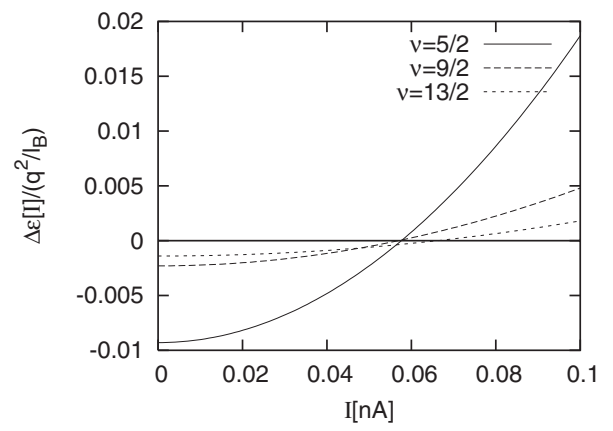

FIG. 10. Energy differences $\Delta E[I]$ between the striped Hall state and the ACDW state. The results at $\nu=5 / 2,9 / 2,13 / 2$ are shown. When $\Delta \epsilon[I]$ is positive, the striped Hall state has a lower energy. charge accumulation results in the energy enhancement via the Coulomb interaction between the accumulated charges. The energy enhancement was estimated from the current and charge distributions. It is found that the energy of the ACDW state increases faster than that of the striped Hall state does as the injected current increases. In the system with no injected current, the energy of the ACDW state is lower than that of the striped Hall state. Hence, the striped Hall state becomes the lower energy state when the current exceeds the critical value. The critical value is estimated at about 0.04-0.07 nA. The current used in the experiments for the anisotropic states $^{6,7}$ is above $1 \mathrm{nA}$. This result suggests that the striped Hall state is realized in the experiments. In addition, the striped Hall state has the anisotropic Fermi surface, which naively explains the experimental features of the anisotropic states, i.e., the anisotropic longitudinal resistivities and the unquantized Hall resistivities. Hence, we conclude that the striped Hall state is realized in the experiment rather than the ACDW state and predict that the ACDW state is realized if the experiment is done with the current smaller than the critical value.

\section{ACKNOWLEDGMENTS}

This work was partially supported by the special Grantin-Aid for Promotion of Education and Science in Hokkaido University, a Grant-in-Aid for Scientific Research on Priority Area (Dynamics of Superstrings and Field Theories, Grant No. 13135201), and (Progress in Elementary Particle Physics of the 21st Century through Discoveries of Higgs Boson and Supersymmetry, Grant No. 16081201), provided by the Ministry of Education, Culture, Sports, Science, and Technology, Japan.

\section{APPENDIX A: HARTREE-FOCK HAMILTONIAN}

Let us consider the quantum Hall system and concentrate on the Coulomb interaction part of the Hamiltonian. The Coulomb interaction part is given by

$$
H_{\text {int }}=\frac{1}{2} \int \frac{d^{2} k}{(2 \pi)^{2}}: \rho(\mathbf{k}) V(\mathbf{k}) \rho(-\mathbf{k}):
$$

where $\rho(\mathbf{k})$ and $V(\mathbf{k})$ are given in Eqs. (2.10) and (2.11), respectively. The HF approximated form of $H_{\text {int }}$ is written using the vNL base by $H_{\mathrm{HF}}=\mathcal{H}_{\mathrm{HF}}-\left\langle\mathcal{H}_{\mathrm{HF}}\right\rangle / 2$, where

$$
\begin{array}{r}
\mathcal{H}_{\mathrm{HF}}=\sum_{l_{1}, l_{2}, l_{3}, l_{4}} \int \frac{d^{2} k}{(2 \pi)^{2}} v_{l_{1}, l_{2}, l_{3}, l_{4}}^{\mathrm{HF}}(\tilde{\mathbf{k}})\left\langle\bar{\rho}_{l_{1}, l_{2}}(-\tilde{\mathbf{k}})\right\rangle \bar{\rho}_{l_{3}, l_{4}}(\tilde{\mathbf{k}}), \\
\bar{\rho}_{l_{1}, l_{2}}(\mathbf{k})=\int_{\mathrm{BZ}} \frac{d^{2} p}{(2 \pi)^{2}} b_{l_{1}}^{\dagger}(\mathbf{p}) b_{l_{2}}(\mathbf{p}-\hat{\mathbf{k}}) e^{-(i / 4 \pi) \hat{k}_{x}\left(2 p_{y}-\hat{k}_{y}\right)}, \\
v_{l_{1}, l_{2}, l_{3}, l_{4}}^{\mathrm{HF}}(\mathbf{k})=V(\mathbf{k}) f_{l_{1}, l_{2}}^{0}(-\mathbf{k}) f_{l_{3}, l_{4}}^{0}(\mathbf{k})-\int \frac{d^{2} k^{\prime}}{(2 \pi)^{2}} V\left(\mathbf{k}^{\prime}\right) \\
\times f_{l_{1}, l_{4}}^{0}\left(-\mathbf{k}^{\prime}\right) f_{l_{3}, l_{2}}^{0}\left(\mathbf{k}^{\prime}\right) e^{-(i / 2 \pi)\left(k_{x}^{\prime} k_{y}-k_{y}^{\prime} k_{x}\right)} .
\end{array}
$$

$f_{l_{1}, l_{2}}^{0}(\mathbf{k})$ is given in Appendix C. In the definition of the HF potential $v_{l_{1}, l_{2}, l_{3}, l_{4}}^{\mathrm{HF}}(\mathbf{k})$, the first term and the second term in the 
right hand side are the Hartree term and the Fock term, respectively. The LL projected Hamiltonian [Eq. (2.14)] is obtained by projecting $\mathcal{H}_{\mathrm{HF}}$ into the $l$ th LL.

In Sec. IV, the deviations of the magnetic field and the two-point function are taken into account in the long wavelength limit in order to consider the current effect on the HF states. The deviation of the two-point function is caused by the deviation of $\left\langle\bar{\rho}_{l_{1}, l_{2}}(-\tilde{\mathbf{k}})\right\rangle$. We only consider the deviation at the partially filled LL $l_{0}$ since it would give the largest contribution in our calculation and denote it as $\delta \bar{\rho}_{l_{0}}(-\tilde{\mathbf{k}})$. Then, the deviation of $\mathcal{H}_{\mathrm{HF}}$ is given by

$$
\delta \mathcal{H}_{\mathrm{HF}}=\sum_{l_{3}, l_{4}} \int \frac{d^{2} k}{(2 \pi)^{2}} v_{l_{0}, l_{0}, l_{3}, l_{4}}^{\mathrm{HF}}(\widetilde{\mathbf{k}}) \delta \bar{\rho}_{l_{0}}(-\widetilde{\mathbf{k}}) \bar{\rho}_{l_{3}, l_{4}}(\widetilde{\mathbf{k}}) .
$$

In the long wavelength limit, $\delta \bar{\rho}_{l_{0}}(-\widetilde{\mathbf{k}})$ is relevant only for the small momentum. When we expand $v_{l_{1}, l_{2}, l_{3}, l_{4}}^{\mathrm{HF}}(\widetilde{\mathbf{k}})$ with respect to $\mathbf{k}$, the largest contribution comes from the lowest order term with respect to $\mathbf{k}$. For each set of the LLs $\left(l_{3}, l_{4}\right)$, the Hartree term of the HF potential gives the lower order term with respect to $\mathbf{k}$ since the Hartree term has $V(\mathbf{k})$, which is $\mathcal{O}(1 / k)$. Hence, the Hartree term gives the main contribution, and the Fock term is negligible in the long wavelength limit.

\section{APPENDIX B: SELF-CONSISTENT EQUATION FOR THE ANISOTROPIC CHARGE DENSITY WAVE STATE}

The ACDW state at $\nu^{*}=1 / 2$ is constructed using the mean value of the projected density operator given by Eq. (2.28). From Eq. (2.28), the two-point function of the operator $b_{l}(\mathbf{p})$ is obtained by

$$
\begin{aligned}
\left\langle b_{l}^{\dagger}(\mathbf{p}) b_{l^{\prime}}\left(\mathbf{p}^{\prime}\right)\right\rangle_{\mathrm{ACDW}}= & \delta_{l, l^{\prime}} \sum_{\mathbf{N}} e^{-i \phi(\mathbf{p}, \mathbf{N})}\left[F _ { 0 } ( \mathbf { p } ) ( 2 \pi ) ^ { 2 } \delta ^ { 2 } \left(\mathbf{p}-\mathbf{p}^{\prime}\right.\right. \\
& -2 \pi \mathbf{N})+F_{1}(\mathbf{p})(2 \pi)^{2} \delta\left(p_{x}-p_{x}^{\prime}\right. \\
& \left.\left.-2 \pi N_{x}\right) \delta\left(p_{y}-p_{y}^{\prime}-\pi\left(2 N_{y}+1\right)\right)\right],
\end{aligned}
$$

where

$$
\begin{aligned}
F_{0}(\mathbf{p})=\sum_{\mathbf{N}} \Delta_{l}( & \left.\frac{2 \pi N_{x}}{r_{s}}, 2 \pi N_{y} r_{s}\right) e^{-i p_{x} N_{y}+i p_{y} N_{x}-i \pi\left(N_{x}+N_{y}+N_{x} N_{y}\right)}, \\
F_{1}(\mathbf{p})= & \sum_{\mathbf{N}} \Delta_{l}\left[\frac{2 \pi N_{x}}{r_{s}}, \pi\left(2 N_{y}+1\right) r_{s}\right] \\
& \times e^{-i p_{x} N_{y}+i p_{y} N_{x}-i \pi\left[N_{x}+N_{y}+N_{x}\left(N_{y}+1 / 2\right)\right]} .
\end{aligned}
$$

The HF Hamiltonian of the ACDW state is given by Eq. (2.31), and the ground state is the state in which the lower energy band at the uppermost partially filled LL $l$ is fully occupied. The ground state is expressed in terms of the field operator $c_{-}(\mathbf{p})$ by $|\Omega\rangle=N_{c} \Pi_{\mathbf{p} \in \mathrm{RBZ}} c_{-}^{\dagger}(\mathbf{p})|0\rangle$, where $N_{c}$ is a normalization constant and $|0\rangle$ is a vacuum state in which the $(l-1)$ th and lower Landau levels are fully occupied. The self-consisntent equation for $\Delta_{l}\left(\mathbf{Q}_{N}\right)$ is obtained by calculating the the left hand side of Eq. (B1) for this ground state.

Assuming the $x$ - and $y$-inversion symmetries for the density, the order parameters become real and have the property $\Delta_{l}\left(Q_{x}, Q_{y}\right)=\Delta_{l}\left(Q_{x},-Q_{y}\right)=\Delta_{l}\left(-Q_{x}, Q_{y}\right)$. The selfconsistent solution is available only when the two energy bands are symmetric with respect to the energy gap i.e., $\epsilon_{+}(\mathbf{p})=-\epsilon_{-}(\mathbf{p}) \equiv \epsilon(\mathbf{p})$, as expected from the particle-hole symmetry of the original Hamiltonian. This gives $\operatorname{Tr}_{2 \times 2} D_{l}(\mathbf{p})=0$, where $\operatorname{Tr}_{2 \times 2}$ denotes the trace with respect to the $2 \times 2$ matrix indices, and $A\left(p_{x}, p_{y}+\pi\right)=-A(\mathbf{p})$. In this case, $U(\mathbf{p})$ and $\epsilon(\mathbf{p})$ are given by

$$
\begin{gathered}
U(\mathbf{p})=\left(\begin{array}{cc}
\frac{B(\mathbf{p})}{N_{+}(\mathbf{p})} & \frac{B(\mathbf{p})}{N_{-}(\mathbf{p})} \\
\frac{\boldsymbol{\epsilon}_{+}(\mathbf{p})-A(\mathbf{p})}{N_{+}(\mathbf{p})} & \frac{\boldsymbol{\epsilon}_{-}(\mathbf{p})-A(\mathbf{p})}{N_{-}(\mathbf{p})}
\end{array}\right), \\
\epsilon(\mathbf{p})=\sqrt{[A(\mathbf{p})]^{2}+|B(\mathbf{p})|^{2}},
\end{gathered}
$$

where $N_{ \pm}(\mathbf{p})=2 \epsilon_{ \pm}(\mathbf{p})\left[\epsilon_{ \pm}(\mathbf{p})-A(\mathbf{p})\right]$.

\section{APPENDIX C: LANDAU LEVEL MATRIX ELEMENTS}

The matrix elements $\left\langle l_{1}\left|e^{i \boldsymbol{q} \cdot \xi}\right| l_{2}\right\rangle$ are given as follows:

$$
\begin{aligned}
& \left\langle l_{1}\left|e^{i \mathbf{q} \cdot \xi}\right| l_{2}\right\rangle \\
& = \begin{cases}\sqrt{\frac{l_{1} !}{l_{2} !}}\left(\frac{q_{x}+i q_{y}}{\sqrt{4 \pi}}\right)^{l_{2}-l_{1}} e^{-q^{2} / 8 \pi} L_{l_{1}}^{l_{2}-l_{1}}\left(\frac{q^{2}}{4 \pi}\right) & \text { for } l_{2}>l_{1} \\
\sqrt{\frac{l_{2} !}{l_{1} !}}\left(\frac{q_{x}-i q_{y}}{\sqrt{4 \pi}}\right)^{l_{1}-l_{2}} e^{-q^{2} / 8 \pi} L_{l_{2}}^{l_{1}-l_{2}}\left(\frac{q^{2}}{4 \pi}\right) & \text { for } l_{1}>l_{2} \\
e^{-q^{2} / 8 \pi} L_{l_{1}}\left(\frac{q^{2}}{4 \pi}\right) & \text { for } l_{2}=l_{1} .\end{cases}
\end{aligned}
$$

From Eq. (C1), we obtain $f_{l_{1}, l_{2}}^{\mu}(\mathbf{q})$ defined by Eq. (3.3) as $f_{l_{1}, l_{2}}^{0}(\mathbf{q})=\left\langle l_{1}\left|e^{i \mathbf{q} \cdot \boldsymbol{\xi}}\right| l_{2}\right\rangle, f_{l_{1}, l_{2}}^{x}(\mathbf{q})=i \omega_{c} \partial_{q_{y}}\left\langle l_{1}\left|e^{i \mathbf{q} \cdot \boldsymbol{\xi}}\right| l_{2}\right\rangle$, and $f_{l_{1}, l_{2}}^{y}(\mathbf{q})$ $=-i \omega_{c} \partial_{q_{x}}\left\langle l_{1}\left|e^{i \mathbf{q} \cdot \xi}\right| l_{2}\right\rangle$. Note that $\left\{f_{l_{1}, l_{2}}^{\mu}(-k)\right\}^{*}=f_{l_{2}, l_{1}}^{\mu}(k)$ holds following from its definition. The values of $f_{l_{1}, l_{2}}^{\mu}(0)$ and its derivatives are given as

$$
\begin{gathered}
f_{l_{1}, l_{2}}^{0}(0)=\delta_{l_{1}, l_{2},} \\
f_{l_{1}, l_{2}}^{x}(0)=\left.i \omega_{c} \frac{\partial f_{l_{1}, l_{2}}^{0}(q)}{\partial q_{y}}\right|_{q=0} \\
= \begin{cases}-\omega_{c} \sqrt{\frac{l_{1}+1}{4 \pi}} \delta_{l_{2}, l_{1}+1} & \text { for } l_{2}>l_{1} \\
\omega_{c} \sqrt{\frac{l_{1}}{4 \pi}} \delta_{l_{1}, l_{2}+1} & \text { for } l_{1}>l_{2} \\
0 & \text { for } l_{1}=l_{2},\end{cases}
\end{gathered}
$$




$$
\begin{aligned}
f_{l_{1}, l_{2}}^{y}(0)= & -\left.i \omega_{c} \frac{\partial f_{l_{1}, l_{2}}^{0}(q)}{\partial q_{x}}\right|_{q=0} \\
& = \begin{cases}-i \omega_{c} \sqrt{\frac{l_{1}+1}{4 \pi}} \delta_{l_{2}, l_{1}+1} & \text { for } l_{2}>l_{1} \\
-i \omega_{c} \sqrt{\frac{l_{1}}{4 \pi}} \delta_{l_{1}, l_{2}+1} & \text { for } l_{1}>l_{2} \quad(\mathrm{C} 3) \\
0 & \text { for } l_{1}=l_{2},\end{cases} \\
& = \begin{cases}-i \frac{\omega_{c}}{4 \pi} \sqrt{\left(l_{1}+1\right)\left(l_{1}+2\right)} \delta_{l_{2}, l_{1}+2}(q) & \text { for } l_{2}>l_{1} \\
-i \frac{\omega_{c}}{4 \pi} \sqrt{l_{1}\left(l_{1}-1\right)} \delta_{l_{1}, l_{2}+2} & \text { for } l_{1}>l_{2} \\
-i \frac{\omega_{c}}{4 \pi}\left(l_{1}+\frac{1}{2}\right) & \text { for } l_{1}=l_{2},\end{cases} \\
& = \begin{cases}-i \frac{\omega_{c}}{4 \pi} \sqrt{l_{1}\left(l_{1}-1\right)} \delta_{l_{1}, l_{2}+2} & \text { for } l_{1}>l_{2} \\
i \frac{\omega_{c}}{4 \pi}\left(l_{1}+\frac{1}{2}\right) & \text { for } l_{1}=l_{2} .\end{cases} \\
-i \frac{\omega_{c}}{4 \pi} \sqrt{\left(l_{1}+1\right)\left(l_{1}+2\right)} \delta_{l_{2}, l_{1}+2} & \text { for } l_{2}>l_{1}
\end{aligned}
$$

(C5)

\section{APPENDIX D: CALCULATION OF $K^{00}$ \\ FOR THE ANISOTROPY CHARGE \\ DENSITY WAVE STATE}

When $p_{0}=p_{y}=0$ and $p_{x} \rightarrow 0$, the response function $K_{0}^{00}\left(p_{x}\right)$ is Taylor expanded with respect to $p_{x}$ as

$$
K_{0}^{00}\left(p_{x}\right)=K_{0}^{00}(0)+p_{x} \partial_{p_{x}} K_{0}^{00}(0)+\frac{p_{x}^{2}}{2} \partial_{p_{x}}^{2} K_{0}^{00}(0)+\cdots
$$

The first and second terms become zero. The third term includes the corrections from the inter-LL term and the intra-LL term. The inter-LL term gives the same expression for $K_{0}^{00}$ as that in the striped Hall state. The intra-LL term gives the extra correction $\Delta K_{0}^{00}\left(p_{x}\right)$ given by

$$
\Delta K_{0}^{00}\left(p_{x}\right)=\kappa p_{x}^{2},
$$

where $\kappa$ is given by

$$
\begin{aligned}
\kappa= & \frac{e^{2}}{2} \int_{\mathrm{RBZ}} \frac{d^{2} p^{\prime}}{(2 \pi)^{2}} \frac{1}{2 \epsilon\left(\mathbf{p}^{\prime}\right)} \partial_{p_{x}}^{2} \\
& \times\left.\left\{\frac{A\left(\hat{\mathbf{p}}+\mathbf{p}^{\prime}\right) A\left(\mathbf{p}^{\prime}\right)+\operatorname{Re}\left[B\left(\hat{\mathbf{p}}+\mathbf{p}^{\prime}\right) B^{*}\left(\mathbf{p}^{\prime}\right) e^{-(i / 2) \hat{p}_{x}}\right]}{\epsilon\left(\hat{\mathbf{p}}+\mathbf{p}^{\prime}\right) \epsilon\left(\mathbf{p}^{\prime}\right)}\right\}\right|_{p_{x}=0} .
\end{aligned}
$$

$\beta$ in Eq. (3.23) is defined by $\beta \equiv-\kappa \nu \omega_{c} / \sigma_{x y}^{(\nu)} \sqrt{B}$. The finite $\kappa$ is the result of the band formation at the partiall filled LL, while the band structure is generated by the density modulation of the ACDW state in both directions. Hence, it may be considered that the finite $\kappa$ reflects the remaining density modulation effect of the ACDW state in the long wavelength limit.
${ }^{1}$ J. K. Jain, Phys. Rev. Lett. 63, 199 (1989).

${ }^{2}$ B. I. Halperin, P. A. Lee, and N. Read, Phys. Rev. B 47, 7312 (1993); Composite Fermions: A Unified View of the Quantum Hall Regime, edited by O. Heinonen (World Scientific, Singapore, 1998).

${ }^{3}$ R. L. Willett, J. P. Eisenstein, H. L. Stormer, D. C. Tsui, A. C. Gossard, and J. H. English, Phys. Rev. Lett. 59, 1776 (1987); W. Pan, J.-S. Xia, V. Shvarts, D. E. Adams, H. L. Stormer, D. C. Tsui, L. N. Pfeiffer, K. W. Baldwin, and K. W. West, ibid. 83, 3530 (1999).

${ }^{4}$ G. Moore and N. Read, Nucl. Phys. B 360, 362 (1991).

${ }^{5}$ M. Greiter, X.-G. Wen, and F. Wilczek, Phys. Rev. Lett. 66, 3205 (1991).

${ }^{6}$ M. P. Lilly, K. B. Cooper, J. P. Eisenstein, L. N. Pfeiffer, and K. W. West, Phys. Rev. Lett. 82, 394 (1999).

${ }^{7}$ R. R. Du, D. C. Tsui, H. L. Stormer, L. N. Pfeiffer, K. W. Baldwin, and K. W. West, Solid State Commun. 109, 389 (1999).

${ }^{8}$ A. A. Koulakov, M. M. Fogler, and B. I. Shklovskii, Phys. Rev. Lett. 76, 499 (1996); M. M. Fogler, A. A. Koulakov, and B. I.
Shklovskii, Phys. Rev. B 54, 1853 (1996).

${ }^{9}$ R. Moessner and J. T. Chalker, Phys. Rev. B 54, 5006 (1996).

${ }^{10}$ E. Fradkin and S. A. Kivelson, Phys. Rev. B 59, 8065 (1999).

${ }^{11}$ E. H. Rezayi, F. D. M. Haldane, and K. Yang, Phys. Rev. Lett. 83, 1219 (1999).

${ }^{12}$ A. H. MacDonald and M. P. A. Fisher, Phys. Rev. B 61, 5724 (2000).

${ }^{13}$ M. M. Fogler and V. M. Vinokur, Phys. Rev. Lett. 84, 5828 (2000).

${ }^{14}$ C. Wexler and A. T. Dorsey, Phys. Rev. B 64, 115312 (2001).

${ }^{15}$ A. Lopatnikova, S. H. Simon, B. I. Halperin, and X.-G. Wen, Phys. Rev. B 64, 155301 (2001).

${ }^{16}$ V. Oganesyan, S. A. Kivelson, and E. Fradkin, Phys. Rev. B 64, 195109 (2001).

${ }^{17}$ N. Shibata and D. Yoshioka, Phys. Rev. Lett. 86, 5755 (2001).

${ }^{18}$ T. Aoyama, K. Ishikawa, Y. Ishizuka, and N. Maeda, Phys. Rev. B 66, 155319 (2002); 70, 035314 (2004).

${ }^{19}$ R. Côté and H. A. Fertig, Phys. Rev. B 62, 1993 (2000).

${ }^{20}$ K. Ishikawa, N. Maeda, and T. Ochiai, Phys. Rev. Lett. 82, 4292 
(1999).

${ }^{21}$ N. Maeda, Phys. Rev. B 61, 4766 (2000).

${ }^{22}$ D. Yoshioka and H. Fukuyama, J. Phys. Soc. Jpn. 47, 394 (1979); D. Yoshioka and P. A. Lee, Phys. Rev. B 27, 4986 (1983).

${ }^{23}$ A. H. MacDonald, T. M. Rice, and W. F. Brinkman, Phys. Rev. B 28, 3648 (1983).

${ }^{24}$ D. J. Thouless, J. Phys. C 18, 6211 (1985).

${ }^{25}$ P. F. Fontein, J. A. Kleinen, P. Hendriks, F. A. P. Blom, J. H. Wolter, H. G. M. Lochs, F. A. J. M. Driessen, L. J. Giling, and C. W. J. Beenakker, Phys. Rev. B 43, 12090 (1991).

${ }^{26}$ C. W. J. Beenakker and H. van Houten, in Solid State Physics, edited by H. Ehrenreich and D. Turnbull (Academic, New York,
1992), Vol. 44, pp. 177-181.

${ }^{27}$ A. M. Perelomov, Teor. Mat. Fiz. 6, 213 (1971).

${ }^{28}$ V. Bargmann, P. Butera, L. Girardello, and J. R. Klauder, Rep. Math. Phys. 2, 221 (1971).

${ }^{29}$ N. Imai, K. Ishikawa, T. Matsuyama, and I. Tanaka, Phys. Rev. B 42, 10610 (1990); K. Ishikawa, N. Maeda, T. Ochiai, and H. Suzuki, Physica E (Amsterdam) 4, 37 (1999).

${ }^{30}$ A. H. MacDonald and S. M. Girvin, Phys. Rev. B 38, 6295 (1988).

${ }^{31}$ M. O. Goerbig, P. Lederer, and C. M. Smith, Phys. Rev. B 69, 115327 (2004). 